\title{
Increasing the Fungicidal Action of Amphotericin B by Inhibiting the Nitric Oxide-Dependent Tolerance Pathway
}

\author{
Kim Vriens, ${ }^{1}$ Phalguni Tewari Kumar, ${ }^{2}$ Caroline Struyfs, ${ }^{1,3}$ Tanne L. Cools, ${ }^{1}$ \\ Pieter Spincemaille, ${ }^{4}$ Tadej Kokalj, ${ }^{2}$ Belém Sampaio-Marques, ${ }^{5,6}$ Paula Ludovico, ${ }^{5,6}$ \\ Jeroen Lammertyn, ${ }^{2}$ Bruno P. A. Cammue, ${ }^{1,3}$ and Karin Thevissen ${ }^{1}$ \\ ${ }^{1}$ Centre of Microbial and Plant Genetics, KU Leuven, 3001 Heverlee, Belgium \\ ${ }^{2}$ BIOSYST-MEBIOS, KU Leuven, 3001 Heverlee, Belgium \\ ${ }^{3}$ VIB Department of Plant Systems Biology, 9052 Ghent, Belgium \\ ${ }^{4}$ Department of Laboratory Medicine, University Hospitals Leuven, Herestraat 49, 3000 Leuven, Belgium \\ ${ }^{5}$ Life and Health Sciences Research Institute (ICVS), School of Health Sciences, University of Minho, Braga, Portugal \\ ${ }^{6}$ ICVS/3B's-PT Government Associate Laboratory, Braga/Guimarães, Portugal
}

Correspondence should be addressed to Bruno P. A. Cammue; bruno.cammue@biw.kuleuven.be

Received 18 May 2017; Accepted 2 August 2017; Published 10 October 2017

Academic Editor: Reiko Matsui

Copyright ( 2017 Kim Vriens et al. This is an open access article distributed under the Creative Commons Attribution License, which permits unrestricted use, distribution, and reproduction in any medium, provided the original work is properly cited.

Amphotericin $\mathrm{B}(\mathrm{AmB})$ induces oxidative and nitrosative stresses, characterized by production of reactive oxygen and nitrogen species, in fungi. Yet, how these toxic species contribute to AmB-induced fungal cell death is unclear. We investigated the role of superoxide and nitric oxide radicals in AmB's fungicidal activity in Saccharomyces cerevisiae, using a digital microfluidic platform, which enabled monitoring individual cells at a spatiotemporal resolution, and plating assays. The nitric oxide synthase inhibitor L-NAME was used to interfere with nitric oxide radical production. L-NAME increased and accelerated AmB-induced accumulation of superoxide radicals, membrane permeabilization, and loss of proliferative capacity in S. cerevisiae. In contrast, the nitric oxide donor S-nitrosoglutathione inhibited AmB's action. Hence, superoxide radicals were important for AmB's fungicidal action, whereas nitric oxide radicals mediated tolerance towards AmB. Finally, also the human pathogens Candida albicans and Candida glabrata were more susceptible to AmB in the presence of L-NAME, pointing to the potential of AmB-LNAME combination therapy to treat fungal infections.

\section{Introduction}

Pathogenic fungi, including Candida albicans and Candida glabrata, encounter diverse environmental stresses when colonizing human tissues. During the infection process, they are exposed to potent reactive oxygen and nitrogen species (ROS and RNS, resp.), including nitric oxide radical $\left(\mathrm{NO}^{\circ}\right)$, peroxynitrite $\left(\mathrm{ONOO}^{-}\right)$, superoxide anion radical $\left(\mathrm{O}_{2}^{-\bullet}\right)$, and hydroxyl radical $\left({ }^{\bullet} \mathrm{OH}\right)$, generated by the respiratory burst of phagocytic cells [1-3]. ROS and RNS cause damage to DNA, proteins, and lipids and are toxic to most fungi $[4,5]$. In contrast to most nonpathogenic fungi, fungal pathogens such as several Candida species have developed responses to neutralize these toxic radicals and repair the potential molecular damage [6]. In this respect, various proteins that protect the fungus from oxidative and nitrosative stresses have been identified and include signalling proteins, transcription factors, and a variety of other enzymes such as catalases, superoxide dismutases, peroxidases, and nitric oxide dioxygenase $[1,7]$. Hence, antifungals (or combinations thereof) inducing an excess ROS and/or RNS in a pathogenic fungus that cannot be neutralized by its endogenous protection mechanisms are of great interest [8].

Many antifungal agents are reported to induce oxidative (excess ROS) stress in pathogenic fungi. These agents include small molecules, such as miconazole $[9,10]$, fluconazole $[11,12]$, amphotericin $B(\mathrm{AmB})[12-16]$, and caspofungin [17], but also antimicrobial peptides, such as protonectin 
[18], baicalin [19], and various plant defensins [20-23]. To date, the induction of nitrosative (excess RNS) stress in fungal species has only been demonstrated for AmB in the pathogenic fungus Cryptococcus gattii [24] and for the plant defensins $\mathrm{NaD} 1$ and PvD1 in C. albicans [23, 25]. AmB belongs to the polyene class of antifungals and induces fungal cell death through apoptotic and nonapoptotic pathways [26-29]. Hence, based on the above reports, it seems that $\mathrm{AmB}$ can induce both excess ROS and RNS in pathogenic fungi. How the production of these different types of radicals would contribute to AmB's fungicidal action is hitherto not known. Moreover, increased insight in these AmB-induced events may lead to more efficient AmB-based therapies, as exemplified in the current study.

In this study, we further investigated the potential of AmB to induce ROS and RNS and looked at the interplay between these toxic radicals and their accumulation kinetics, thereby linking these events to AmB's killing capacity. To investigate the kinetics of the AmB-induced ROS and RNS, we used a digital microfluidic platform (DMF) in which single cells were captured and monitored over time using time lapse fluorescence microscopy. This DMF platform has been previously optimized for seeding of Saccharomyces cerevisiae cells and subsequently for assessing the rate by which AmBinduced membrane permeabilization events occurred at the single cell level [30]. S. cerevisiae has been widely used to investigate the mechanisms of action of antifungal agents, including that of $\mathrm{AmB}[14,31-35]$. Hence, also in this study, we used $S$. cerevisiae as a model organism to better understand the mode of action of $\mathrm{AmB}$ and translated the most prominent findings to the fungal pathogens C. albicans and C. glabrata.

\section{Methods}

2.1. Strains and Chemical Reagents. Saccharomyces cerevisiae strain BY4741, Candida albicans strain SC5314, and Candida glabrata strain BG2 were used in the cytotoxicity assays. All culture media were purchased from LabM Ltd. (Lancashire, England), unless stated otherwise. Media used were YPD ( $1 \%$ yeast extract; $2 \%$ peptone; and $2 \%$ glucose), $1 / 5$ YPD (YPD diluted in distilled water), and RPMI-1640 (Roswell Park Memorial Institute-1640 medium; pH7) with Lglutamine and without sodium bicarbonate (purchased from Sigma-Aldrich, St. Louis, MO, USA), buffered with MOPS (Sigma-Aldrich, St. Louis, MO, USA).

Amphotericin B (AmB), $N_{\omega}$-Nitro-L-arginine methyl ester hydrochloride (L-NAME), S-nitrosoglutathione, propidium iodide (PI), and dihydroethidium (DHE) were purchased from Sigma-Aldrich (St. Louis, MO, USA). 4-Amino-5-methylamino-2', $7^{\prime}$-difluorofluorescein diacetate (DAF-FM DA) was supplied by Life Technologies (Carlsbad, CA, USA). Peroxide was purchased from VWR chemicals (Radnor, PA, USA).

Fluorinert FC-40 was purchased from 3M (St. Paul, $\mathrm{MN}$, USA), and chemicals for photolithopgraphy were supplied by Rohm and Haas (Marlborough, MN, USA). Fluoroalkylsilane Dynasylan ${ }^{\circledR}$ F 8263 was supplied by Evonik (Essen, Germany). AZ1505 photoresist and Teflon-AF ${ }^{\circledR}$ were obtained from Microchemicals GmbH (Ulm, Germany) and Dupont (Wilmington, DE, USA), respectively. Parylene C dimer and Silane A174 were purchased from Plasma Parylene Coating Services (Rosenheim, Germany).

2.2. Cell Culture Conditions. S. cerevisiae, C. albicans, or $C$. glabrata, grown overnight in YPD at $30^{\circ} \mathrm{C}$ and $250 \mathrm{rpm}$, were diluted to an optical density (OD) of 0.15 measured at $\lambda=600 \mathrm{~nm}$ in a flask containing $50 \mathrm{~mL}$ of fresh YPD and further cultured for $5 \mathrm{~h}$ at $30^{\circ} \mathrm{C}$ and $250 \mathrm{rpm}$ (S. cerevisiae) or $37^{\circ} \mathrm{C}$ and $200 \mathrm{rpm}$ (Candida spp.), to obtain exponentially growing cells. Cells were then pelleted by centrifugation (3 min, $4000 \mathrm{rpm}$ ), washed and resuspended in 1/5 YPD for S. cerevisiae or RPMI-1640 medium for C. albicans and C. glabrata to an OD of 3 for further use in the experiments.

2.3. Cytotoxicity Assays in Bulk. Exponentially growing cells were supplemented with PI, DHE, or DAF-FM DA to a final concentration of $3 \mu \mathrm{M}, 17 \mu \mathrm{M}$, and $5 \mu \mathrm{M}$, respectively, and subsequently treated either with DMSO or water (controls), a range of $\mathrm{AmB}$ dosages $(0.625 \mu \mathrm{M}-10 \mu \mathrm{M}$, dissolved in DMSO), $200 \mathrm{mM}$ L-NAME (dissolved in water), or a combination of the above, with a final DMSO concentration of $1 \%$ $(v / v \%)$. After mixing, the cell suspensions were transferred to Eppendorf tubes, covered with a layer of silicon oil, placed on a horizontal shaker at $5 \mathrm{rpm}$, and incubated in the dark for $3 \mathrm{~h}$ at room temperature to be compliant with the DMF setup. In case of C. albicans and C. glabrata, however, the assays were performed at $37^{\circ} \mathrm{C}$ to be clinically relevant. A plating assay was carried out at the start of the treatment to account for the number of cells at this point. After $3 \mathrm{~h}$, cells were pelleted ( $3 \mathrm{~min}$, $4000 \mathrm{rpm}$ ), washed and resuspended in phosphate buffered saline (PBS), and subsequently plated or subjected to flow cytometry on a BD Influx ${ }^{\mathrm{TM}}$ cell sorter. In the plating assay, a 10-fold dilution series of the cell suspensions was prepared in PBS and appropriate cell suspensions were spread in YPD agar plates, after which the plates were allowed to dry for $10 \mathrm{~min}$ and incubated for $48 \mathrm{~h}$ at $30^{\circ} \mathrm{C}$ to visualize the number of colony forming units (CFUs). For flow cytometry, cells were monitored for fluorescence at 540/608 nm (FL3 610_20), 485/515 nm (FL1 580_30), or $495 / 515 \mathrm{~nm}$ (FL2 530_40) for detection of membrane permeabilization with PI, detection of superoxide radical accumulation with DHE, or detection of nitric oxide radical accumulation with DAF-FM DA, respectively.

2.4. Cell Cycle Analysis. Aliquots of cells were collected at the indicated time points and cells were pelleted, washed, and fixed with ethanol $(70 \% v / v)$ for at least $30 \mathrm{~min}$ at $4^{\circ} \mathrm{C}$. Cells were then resuspended in sodium citrate buffer $(50 \mathrm{mM}$ sodium citrate, $\mathrm{pH} 7.5$ ), sonicated and treated with RNAse for $1 \mathrm{~h}$ at $50^{\circ} \mathrm{C}$, followed by subsequent incubation with $20 \mu \mathrm{g} / \mathrm{ml}$ proteinase $\mathrm{K}$ for 1 hour at $50^{\circ} \mathrm{C}$. Cell DNA was then stained overnight with SYBR Green 10,000 x (Molecular Probes/Invitrogen, Carlsbad, CA), diluted 10-fold in TrisEDTA ( $\mathrm{pH} \mathrm{8.0),} \mathrm{and} \mathrm{incubated} \mathrm{overnight} \mathrm{at} 4^{\circ} \mathrm{C}$. Before flow cytometry analysis, samples were diluted $1: 4$ in sodium citrate buffer. The SYBR signals were measured using a BD LSR II $^{\mathrm{TM}}$ (Becton Dickinson, NJ, USA) with a $488 \mathrm{~nm}$ excitation 
laser. Signals from 30,000 cells/sample were captured in FITC channel $(530 \mathrm{~nm} \pm 30 \mathrm{~nm})$, at a flow rate of about 1000 cells/s. The percentage of cells in each phase of the cell cycle was determined offline with ModFit LT software (Verity Software House, Topsham, ME).

2.5. Checkerboard Antifungal Assays. AmB (dissolved in DMSO), L-NAME (dissolved in water), and Snitrosoglutathione (dissolved in water) were 2 -fold serially diluted across the columns and rows of a 96-well microtiter plate. Subsequently, AmB dilutions were further diluted 10fold in 1/5 YPD. Next, $20 \mu \mathrm{l}$ volumes of these dilutions were transferred to a microtiter plate, allowing the analysis of unique combinations of two compounds. Exponentially growing $S$. cerevisiae cells were diluted to an OD of 0.10 , measured at $\lambda=600 \mathrm{~nm}$, in 1/5 YPD, and subsequently, $80 \mu \mathrm{l}$ was added to the microtiter plate, resulting in a final DMSO concentration of $1 \%(v / v \%)$. In parallel, the number of colony forming units (CFU) of this exponential culture was determined by plating assay. After $24 \mathrm{~h}$ of incubation at $30^{\circ} \mathrm{C}$, the $\mathrm{OD}$ of the checkerboard plate was measured at $\lambda=600 \mathrm{~nm}$, to examine the growth of $S$. cerevisiae cells. Subsequently, a plating assay was performed of specific wells of the checkerboard assay to assess cell viability of specific AmB-L-NAME combinations. Based on preliminary data of this experiment, we have performed power calculations $(\alpha=0.05 ; b=0.8)$ for an AmB concentration of $0.313 \mu \mathrm{M}$, which is the tested value closest to the IC50 $(0.282 \mu \mathrm{M}$ $\mathrm{AmB}$ for $S$. cerevisiae). These calculations indicated that we needed 2 biological replicates to assure a power of $b=0.8$. Recalculations of the power calculations, based on data of the 2 biologically independent experiments, confirmed the previously executed power calculations.

2.6. Fabrication of Digital Microfluidic Plates. Digital microfluidic plates were fabricated as described previously [30]. The assembly consists of an actuation plate and a grounding plate, as presented in Supplemental Information S3 Figure available online at https://doi.org/10.1155/2017/4064628. For fabricating the actuation plate (S3 Figure A), cleaned glass wafers $(1.1 \mathrm{~mm})$ were sputter coated with chromium $(100 \mathrm{~nm})$ and patterned using standard photolithographic processes. After cleaning the plates with acetone and isopropyl alcohol twice, the surface was plasma activated $\left(\mathrm{O}_{2}\right.$ plasma, 150 mtorr, $\left.100 \mathrm{~W}\right)$ and the plates were primed with Silane A174 to promote adhesion of the parylene C layer $(3 \mu \mathrm{m})$ that was subsequently coated using chemical vapour deposition. Next, a thin layer of Teflon-AF (200 nm, using 3\% $w / w$ in Fluorinert FC-40) was spincoated $(1200 \mathrm{rpm})$ on top of the parylene $\mathrm{C}$ layer and baked for $5 \mathrm{~min}$ at $110^{\circ} \mathrm{C}$ and $5 \mathrm{~min}$ at $200^{\circ} \mathrm{C}$. Crenelated actuation electrodes with dimensions of $2.8 \mathrm{~mm} \times 2.8 \mathrm{~mm}$ were selectively actuated to manipulate individual droplets of $2.7 \mu \mathrm{L}$, using customized software.

For fabrication of the grounding plate (Supplemental Information S3A Figure) of the DMF device, cleaned glass wafers $(1 \mathrm{~mm})$ were coated with an aluminium layer $(40 \mathrm{~nm})$ using thermal evaporation, leaving two $2.5 \times 2.5 \mathrm{~mm}$ visualization windows. Fluoroalkylsilane Dynasylan F 8263 was coated on the aluminium to improve adhesion of the subsequent spin-coated Teflon-AF layer $(3 \mu \mathrm{m})$. Microwells were patterned in the Teflon-AF layer following a hard contact masking procedure, developed by depositing parylene $\mathrm{C}$ $(1 \mu \mathrm{m})$ and aluminium $(60-80 \mathrm{~nm})$ layers. A thin layer of AZ1505 photoresist was spin-coated on the aluminium layer, and the aluminium was patterned and etched using standard photolithography processes. The pattern was then transferred from the aluminum to the Teflon-AF using $\mathrm{O}_{2}$ plasma (150 mtorr, $100 \mathrm{~W}$ ) for $10 \mathrm{~min}$. Finally, using a dry lift-off method, the aluminium-parylene $\mathrm{C}$ mask was peeled off using a pair of forceps, revealing two microwell arrays $(1.9 \mathrm{~mm} \times 1.9 \mathrm{~mm})$ on a single grounding plate, consisting of 22,000 microwells each, arranged in a hexagonal pattern with a pitch distance of $14 \mu \mathrm{m}$ [36]. The dimensions of the microwells were measured to be approximately $5.5 \mu \mathrm{m}$ in width and $3 \mu \mathrm{m}$ in depth.

2.7. Cytotoxicity Assays on DMF Platform. A schematic overview of the cytotoxicity assays on the DMF platform is given in Supplemental Information S3B Figure. Exponentially growing cells were supplemented with PI or DHE to a final concentration of $3 \mu \mathrm{M}$ and $17 \mu \mathrm{M}$, respectively, and subsequently treated either with DMSO or water (controls), a range of $\mathrm{AmB}$ dosages $(5 \mu \mathrm{M}$ and $10 \mu \mathrm{M}$, dissolved in DMSO), $200 \mathrm{mM}$ L-NAME (dissolved in water), or a combination of the above, with a final DMSO concentration of $1 \%$ $(v / v \%)$. After $5 \mathrm{~min}$, two droplets, one containing the mixed cell suspension and one containing the corresponding composition without cells, were placed on two separate electrodes of the actuation plate. The actuation and grounding plate were assembled, thereby aligning the microwell array with the cell droplet and sandwiching it between the plates. To prevent sticking and evaporation of the droplets, $80 \mu \mathrm{L}$ of silicon oil was added between the plates by pipetting. The assembled plates were placed in the DMF chip holder, and the device was flipped upside down and incubated for 10 min to allow sedimentation of the cells. This step was followed by automated shuttling of the cell droplet over the microwell array for 15 times, that is, 15 seeding cycles, using software-assisted electrowetting-on-dielectric (EWOD) actuation. After seeding, the cell droplet was actuated away from the array and a droplet without cells was transferred to the array. The cell responses, that is, membrane permeabilization detected by PI or superoxide radical accumulation detected by $\mathrm{DHE}$, were monitored at room temperature for $3 \mathrm{~h}$ in 15 min intervals using an inverted fluorescence microscope (IX-71, Olympus, Tokyo, Japan) equipped with a CCD camera. The whole array was scanned in 9 overlapping frames in approximately 15 seconds, in which a single frame covered approximately 4100 wells, using a 20x lens magnification. Both fluorescence and bright field images were collected using the same excitation/emission wavelengths as described above.

2.8. Calculation of Fluorescence Intensity per Cell. The DMF array was imaged for $3 \mathrm{~h}$ in $15 \mathrm{~min}$ intervals (i.e., 12 time points), and images were processed in Image $(\mathrm{v} 1.47, \mathrm{NIH}$, $\mathrm{MD}$ ) for background correction using rolling ball algorithm 
with a radius of 50 pixels. Salt-and-pepper noise was removed using the despeckle option in ImageJ. Next, the images were loaded in MATLAB (The Mathworks, Natick, MA), and a custom MATLAB code was executed. The single image captured at $180 \mathrm{~min}$ was processed by MATLAB to identify the single fluorescent cells in contrast with the background. The MATLAB code detected the area of a single cell, and a unique numerical digit was allotted to each cell. Within the detected area of a single cell, the maximum pixel value was registered together with its respective coordinate in a vector array. Next, the MATLAB code was executed on all the images captured between $15 \mathrm{~min}$ and $165 \mathrm{~min}$. The fluorescence intensity of each individual cell in different time frames was monitored by detecting the pixel values for the registered coordinates. The final output was tabular data with pixel intensities of single cells identified with unique numerical digits, as detected in 12 consecutive time points.

2.9. Data Analysis. Flow cytometric data were normalized to the control data, and DMF data were normalized to the first data point, that is, $15 \mathrm{~min}$. For plating assays, the number of CFUs per $\mathrm{mL}$ was displayed in Log scale. Data were analysed with GraphPad Prism 6 SPPS (GraphPad Software, Inc., CA, USA). Two-way ANOVA followed by Dunnett multiple comparison test was performed to analyse statistically significant differences in the number of PI-, DHE-, and DAF-FM DA-positive cells and cells able to proliferate between control and different $\mathrm{AmB}$ treatments in S. cerevisiae, C. albicans, and C. glabrata. Pearson's product-moment correlation was performed to analyse the relation between the results obtained in the bulk and the DMF experiments. Survival analyses (Kaplan-Meier) using the Log-rank test were performed on DMF data to compare survival curves and analyse whether treatment significantly affects survival. Two-way ANOVA and subsequent Tukey or Dunnett multiple comparison tests were performed to analyse differences between bulk results for treatment with $\mathrm{AmB}$ in the presence or absence of L-NAME for each AmB concentration or to analyse differences in bulk results between the first data point and other data points within the same treatment, respectively. Two-way ANOVA followed by Tukey multiple comparison test was performed to analyse statistically significant differences between different treatments in the cell cycle analyses. Two-way ANOVA followed by Tukey multiple comparison test was performed to analyse statistical differences between the OD measurements for treatment with different concentrations of $\mathrm{AmB}$ in the absence or presence of $200 \mathrm{mM}$ L-NAME or $2 \mathrm{mM}$ S-nitrosoglutathione. In all cases, $P<0.05$ was considered statistically significant. When multiple comparisons were performed, multiplicity-adjusted $P$ values for each comparison are presented, taking into account the total number of groups in the ANOVA and the data in all groups.

\section{Results}

3.1. Amphotericin B Induced Nitric Oxide and Superoxide Radical Accumulation in Saccharomyces cerevisiae. First, we assessed whether AmB induces accumulation of superoxide and nitric oxide radicals in $S$. cerevisiae. To this end, yeast cultures were treated with $\mathrm{AmB}$ for $3 \mathrm{~h}$ at room temperature to be compliant with the DMF setup and subjected to flow cytometry to analyse the number of cells with increased superoxide and nitric oxide radical levels using DHE and DAF-FM DA dyes, respectively. The fluorescent probe DHE is typically used for detecting $\mathrm{O}_{2}^{-\bullet}$ due to its relative specificity for this ROS, with minimal oxidation induced by $\mathrm{H}_{2} \mathrm{O}_{2}$ or hypochlorous acid $[37,38]$. Furthermore, in contrast to other intracellular dyes, there is little capacity for the formation of superoxide by DHE due to redox cycling [38]. However, nonspecific oxidation of DHE from other nonsuperoxide sources, such as cytochrome c [38], was not eliminated in this study. The number of cells with compromised plasma membranes was analysed using PI. As PI only enters cells with compromised plasma membrane, it was used as a marker to identify nonapoptotic cell death [39]. To quantify the fungicidal activity of $\mathrm{AmB}$, the treated cultures were subjected to plating assays, thereby assessing the number of cells that was able to proliferate after AmB treatment.

The number of cells that accumulated superoxide (Figure 1(a)) and nitric oxide radicals (Figure 1(b)), as well as the number of cells with permeabilized membranes (Figure 1(c)), was significantly increased by AmB treatment in a dose-dependent manner $(P<0.05)$, while the proliferative capacity of the cells was decreased, yet, not statistically significant (Figure 1(d)). This dose dependency was different for the tested responses: a maximum number of cells producing nitric oxide radicals in AmB-treated yeast cultures were found at $\mathrm{AmB}$ concentrations as low as $2.5 \mu \mathrm{M}(P<0.0001)$ (Figure $1(\mathrm{~b}))$, whereas the highest number of cells with increased superoxide radical accumulation and compromised membranes was observed at $10 \mu \mathrm{M} \mathrm{AmB}$ $(P<0.0001)$ (Figures 1(a) and 1(c), resp.). Hence, it seemed that the production of nitric oxide radicals could be induced at $\mathrm{AmB}$ doses that did not trigger the accumulation of superoxide radicals or membrane permeabilization, while this resulted in a reduced proliferative capacity of cells.

\subsection{Inhibition of Nitric Oxide Radical Production Resulted in} Increased Superoxide Radical Accumulation and Loss of Proliferative Capacity by Amphotericin B. As it was previously shown that superoxide radicals react with nitric oxide radicals, resulting in strongly oxidizing RNS causing damage to proteins and nucleic acids [40-42], we investigated whether the AmB-induced superoxide radical levels could be increased by blocking the production of nitric oxide radicals. To this end, L-NAME was used. L-NAME inhibits nitric oxide synthases in mammalian cells and thus prevents the generation of nitric oxide radicals [43]. Although in yeast, only nitric oxide synthase-like enzymes are identified to date, L-NAME was shown to reduce the levels of nitric oxide radicals in yeast [44-46]. Reduction of the levels of nitric oxide radicals by L-NAME in S. cerevisiae was microscopically confirmed (data not shown).

In the presence of L-NAME, the number of cells with AmB-induced accumulation of superoxide radicals significantly increased as compared to that after AmB treatment 


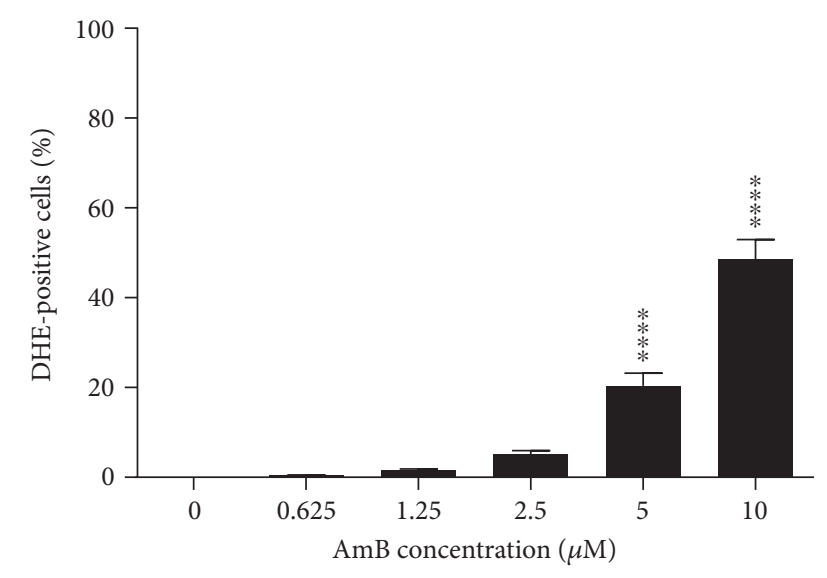

(a)

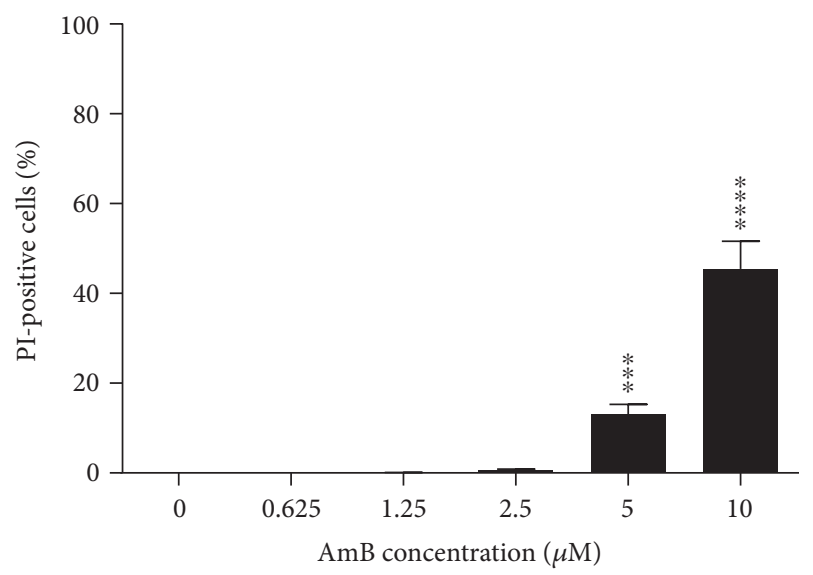

(c)

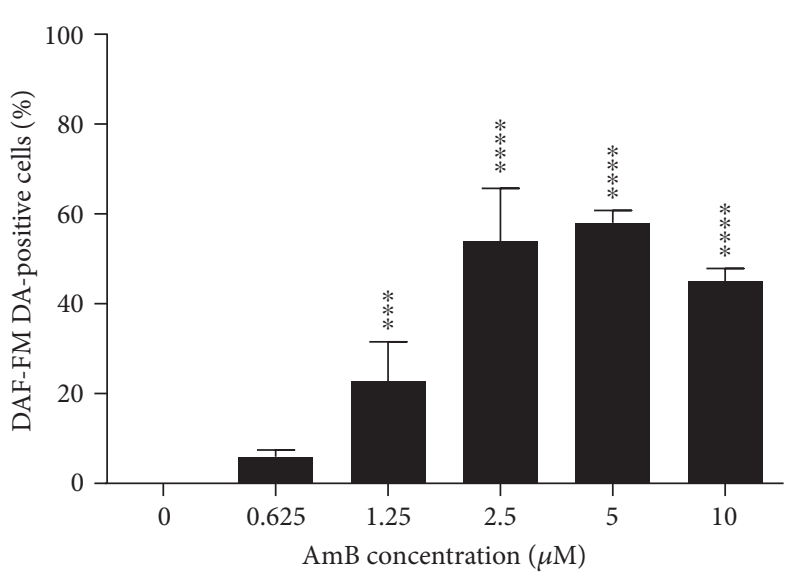

(b)

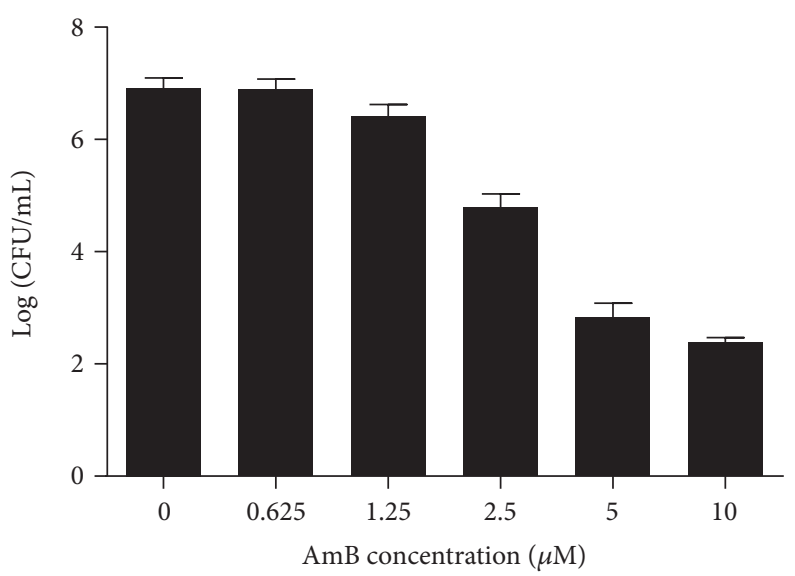

(d)

Figure 1: AmB induced accumulation of superoxide and nitric oxide radicals and membrane permeabilization in S. cerevisiae. Yeast cultures were treated with different concentrations of $\mathrm{AmB}$ for $3 \mathrm{~h}$ and subjected to flow cytometry or plating assays. (a) Levels of superoxide radical detected by dihydroethidium (DHE) fluorescence and flow cytometry. (b) Levels of nitric oxide radical detected by 4-amino-5-methylamino$2^{\prime}, 7^{\prime}$-difluorofluorescein diacetate (DAF-FM DA) fluorescence and flow cytometry. (c) Membrane permeabilization events detected by propidium iodide (PI) fluorescence and flow cytometry. (d) Number of CFU/mL in Log-scale, assessed by plating assays and CFU counting. Means and standard errors of the means (SEM) of at least 3 independent biological experiments $(n \geq 3)$ are presented. Two-way ANOVA followed by Dunnett multiple comparison test was performed to analyse statistically significant differences in the number of PI-, DHE-, and DAF-FM DA-positive cells and cells able to proliferate between control treatment and treatment with different concentrations of AmB. $* * *$ and $* * * *$ represent $P<0.001$ and $P<0.0001$, respectively.

alone, in the case of $2.5 \mu \mathrm{M}$ or $10 \mu \mathrm{M} \mathrm{AmB}$ (Figure 2(a); $P=0.05$ and $P<0.0001$, resp.). Moreover, treatment of yeast with $1.25 \mu \mathrm{M}$ or $2.5 \mu \mathrm{M}$ AmB supplemented with $\mathrm{L}-$ NAME significantly reduced the number of cells that were able to proliferate, as compared to treatment with $\mathrm{AmB}$ alone (Figure 2(c); $P=0.01$ and $P<0.0001$, resp.). In contrast, only $10 \mu \mathrm{M} \mathrm{AmB}$ with L-NAME increased the number of cells with a compromised membrane in a significant manner $(P=0.02)$, as compared to that after treatment with $\mathrm{AmB}$ alone (Figure 2(b)), suggesting that the combination of low concentrations of AmB with $200 \mathrm{mM}$ L-NAME did not affect membrane permeabilization by AmB. In addition, inhibition of nitric oxide radical production resulted in an increased number of cells that accumulated superoxide radicals and was characterized by membrane permeabilization and inability to proliferate. These findings point towards a potential role of nitric oxide radical production in mediating tolerance towards AmB in yeast. Moreover, we have performed cytotoxicity assays with peroxide in the presence and absence of $200 \mathrm{mM}$ L-NAME and found that L-NAME can only increase the killing activity of AmB but not that of peroxide, implying an AmB-specific effect of L-NAME (Supplemental Information S4 Figure).

\subsection{Inhibition of Nitric Oxide Radical Production Resulted in} Faster and Increased Superoxide Radical Accumulation and Faster Membrane Permeabilization by Amphotericin B. To gain more insights into the action of L-NAME on the kinetics of AmB-induced superoxide radical accumulation, time lapse experiments were performed on a DMF platform (for a schematic representation of the experimental setup, see Supplemental Information S3 Figure), as described in our previous study [30]. To this end, yeast was treated with either $0 \mu \mathrm{M}$ (control), $5 \mu \mathrm{M}$, or $10 \mu \mathrm{M} \mathrm{AmB}$ in the presence 


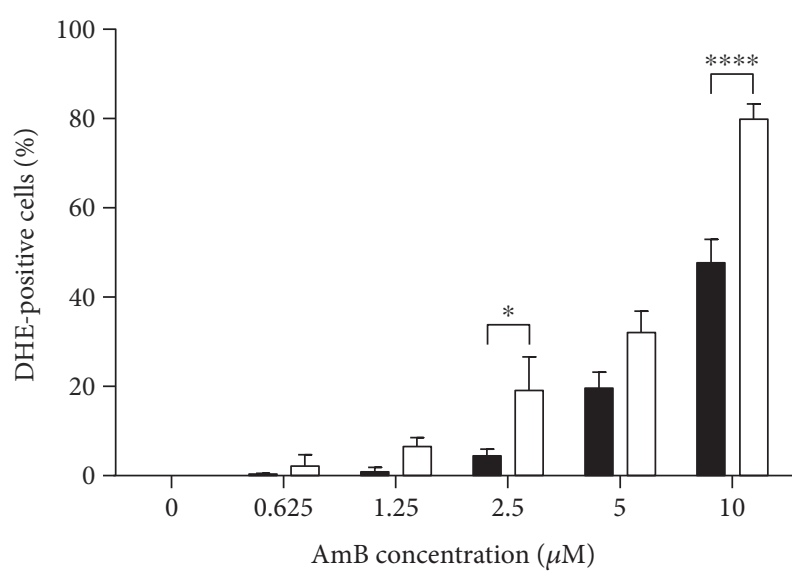

(a)

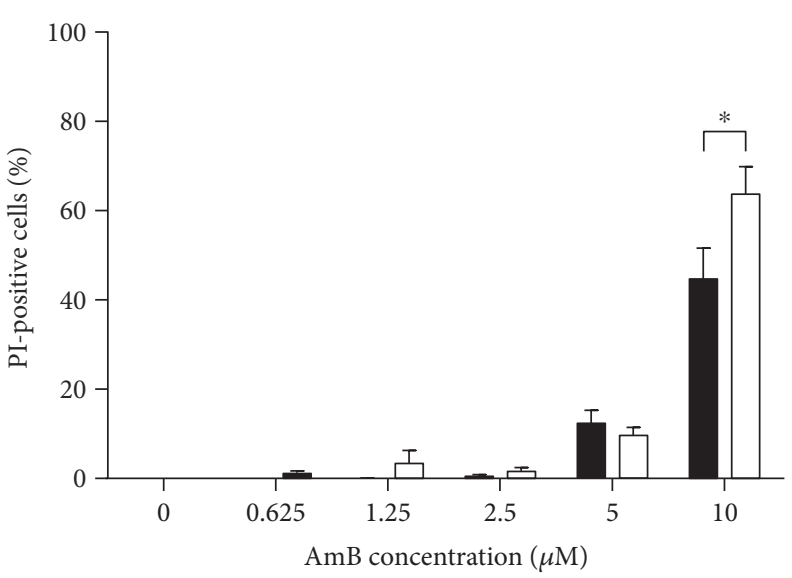

(b)

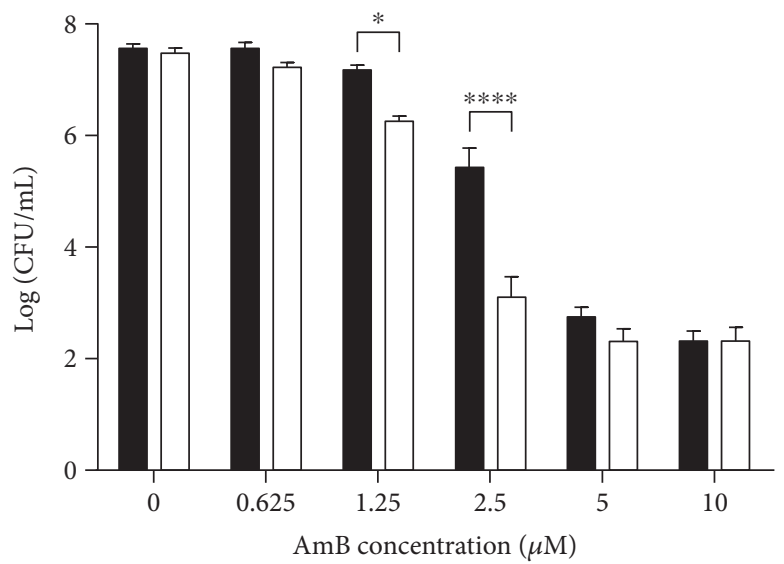

(c)

Figure 2: AmB-induced superoxide radical accumulation, membrane permeabilization, and loss of proliferative capacity can be increased by blocking nitric oxide radical production using L-NAME. Exponential yeast cultures were treated with different concentrations of AmB in the presence or absence of $200 \mathrm{mM}$ L-NAME for $3 \mathrm{~h}$. (a) Levels of superoxide radical detected by dihydroethidium (DHE) fluorescence and flow cytometry. (b) Membrane permeabilization events detected by propidium iodide (PI) fluorescence and flow cytometry. (c) Number of CFU/mL in Log-scale, assessed by plating assays and CFU counting. Means and standard errors of the means (SEMs) of at least 3 independent biological experiments $(n \geq 3)$ are presented. Black bars represent treatment with AmB alone; white bars represent treatment with AmB supplemented with $200 \mathrm{mM}$ L-NAME. Two-way ANOVA followed by Tukey multiple comparison test was performed to analyse significant differences between the two treatments. $*$ and $* * * *$ represent $P<0.05$ and $P<0.0001$, respectively. Multiplicity-adjusted $P$ values are presented in the text.

or absence of $200 \mathrm{mM}$ L-NAME, as these concentrations were shown to have the most profound effect on membrane permeabilization and accumulation of superoxide radicals in the bulk experiments. During treatment, each cell was monitored over time at room temperature for $3 \mathrm{~h}$ in $15 \mathrm{~min}$ intervals for DHE or PI fluorescence. Validation of the DMF platform to monitor DHE and PI fluorescence at single cell level was performed prior to the assays described above (Supplemental Information S1 Figure).

We found an increased number of DHE- (Figure 3(a)) and PI- (Figure 3(b)) positive cells when yeast was treated with $\mathrm{AmB}$ supplemented with $200 \mathrm{mM}$ L-NAME as compared to treatment with $\mathrm{AmB}$ alone, starting from $30 \mathrm{~min}$ to $45 \mathrm{~min}$ incubation, respectively. This observation was in line with the bulk results after $3 \mathrm{~h}$ of incubation at room temperature that were obtained by flow cytometry (Figure 2).
Survival analyses were performed to test the hypothesis that different treatments (i.e., $\mathrm{AmB}$ in the presence or absence of L-NAME) affect survival in a significantly different manner, in which survival is defined as the occurrence of a specific event [47]. Here, we analysed whether the occurrence of superoxide radical accumulation and membrane permeabilization upon treatment with $\mathrm{AmB}$ in the presence and absence of L-NAME was significantly different. The survival curves for treatment with $10 \mu \mathrm{M} \mathrm{AmB}$ in the presence or absence of $200 \mathrm{mM}$ L-NAME were significantly different in both DHE and PI experiments $(P<0.0001)$ (Figures 3(a) and $3(\mathrm{~b})$ ), indicating that L-NAME significantly affected the number of superoxide radical accumulating cells and the number of membrane permeabilization events induced by $\mathrm{AmB}$ over time. When cells were treated with $10 \mu \mathrm{M}$ $\mathrm{AmB}$ in combination with $200 \mathrm{mM}$ L-NAME, a median survival of $45 \mathrm{~min}$ was observed in the DHE experiments, that is, 


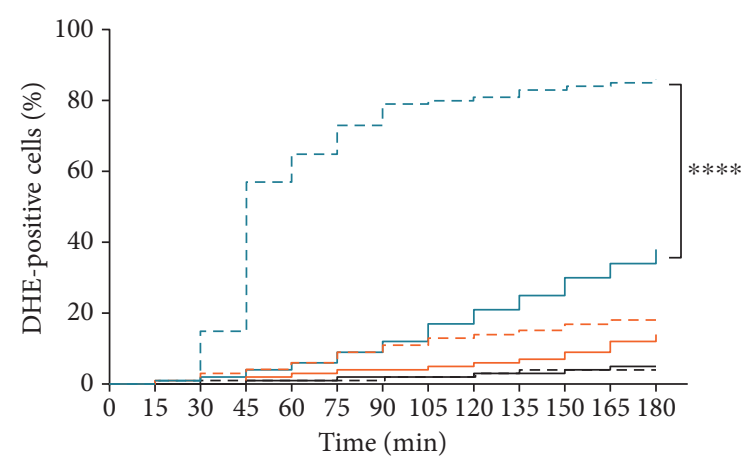

(a)

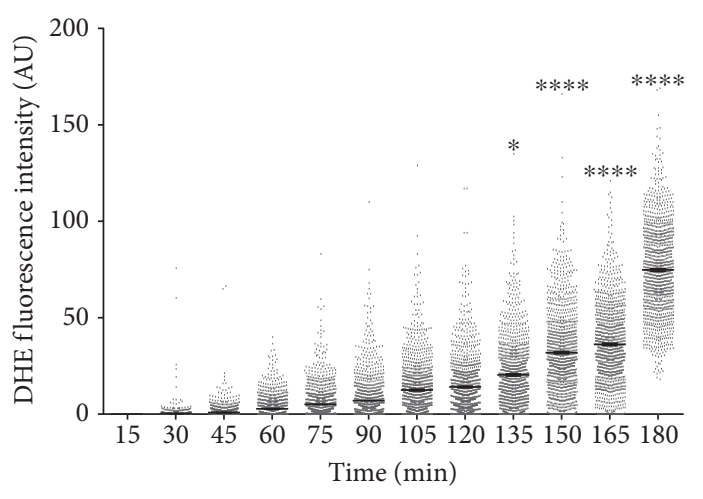

(c)

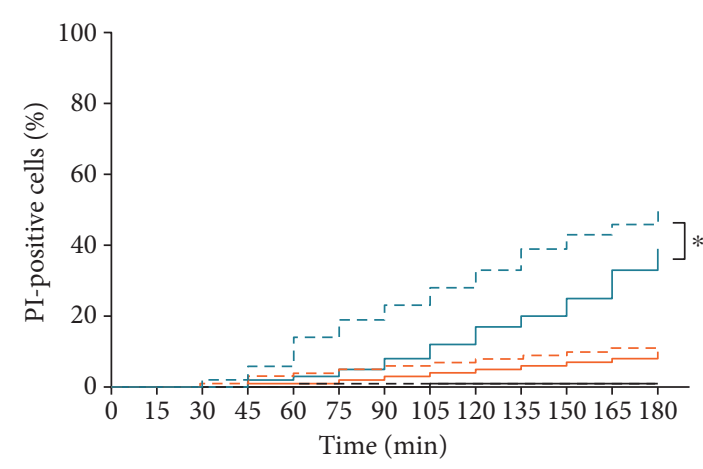

(b)

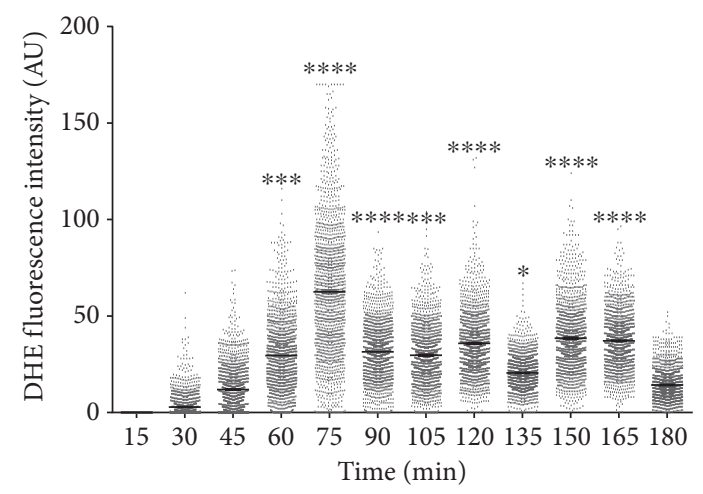

(d)

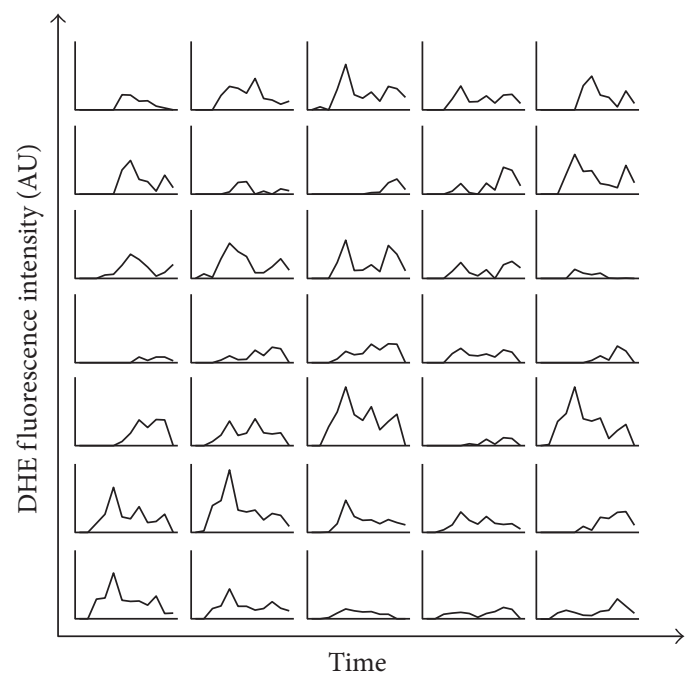

(e)

FIgURE 3: L-NAME increased and accelerated AmB-induced superoxide radical accumulation, membrane permeabilization, and intracellular superoxide radical levels. (a-b) Accumulation of superoxide radicals (a) and membrane permeabilization (b) in S. cerevisiae cells treated either with $0 \mu \mathrm{M}$ (black), $5 \mu \mathrm{M}$ (orange), or $10 \mu \mathrm{M}$ (blue) AmB in the presence (dashed lines) or absence (solid lines) of $200 \mathrm{mM} \mathrm{L-NAME} \mathrm{during} 3 \mathrm{~h}$ in 15 min intervals. Log-rank tests were performed to analyse significant differences between AmB treatment and treatment of AmB in combination with $200 \mathrm{mM}$ L-NAME for each AmB dose. Data of at least 3 independent biological experiments is presented $(n \geq 3)$. $*$ and $* * * *$ represent $P<0.05$ and $P<0.0001$, respectively. (c-d) Intracellular DHE fluorescence in S. cerevisiae cells treated with $10 \mu \mathrm{M}$ AmB in the absence (c) or presence (d) of $200 \mathrm{mM} \mathrm{L-NAME.} \mathrm{Single} \mathrm{cells} \mathrm{were} \mathrm{monitored} \mathrm{for} \mathrm{their} \mathrm{DHE} \mathrm{fluorescence} \mathrm{during} \mathrm{treatment}$ for $3 \mathrm{~h}$ in $15 \mathrm{~min}$ intervals using fluorescence microscopy and the DMF platform. The fluorescence intensity of each cell is presented as arbitrary units (AU), and each dot represents a single cell. Means and standard errors of the means (SEMs) of at least 3 independent biological experiments $(n \geq 3)$, with at least 780 cells each, are presented. Two-way ANOVA followed by Tukey multiple comparison test was performed to analyse significant differences in DHE fluorescence intensity. $*, * * *$, and $* * * *$ represent $P<0.05, P<0.001$, and $P<0.0001$, respectively. (e) DHE fluorescence intensity of individual cells over time. A selection of 35 cells was randomly chosen and is representative for more than 3000 cells that were analysed in this study. Each plot represents the DHE fluorescence intensity, measured every $15 \mathrm{~min}$, of one representative cell over the whole duration of the experiment, that is, $180 \mathrm{~min}$. 
$50 \%$ of the cells was DHE positive after 45 min of treatment. In contrast, when $\mathrm{AmB}$ was applied alone, 50\% of DHEpositive cells in the treated yeast culture was not reached after $180 \mathrm{~min}$ (median survival $>180 \mathrm{~min}$ ), which implied that AmB-induced superoxide radical accumulation occurred faster in cells treated in the presence of L-NAME. Additionally, a hazard ratio (Log-rank) of 4.21 was found when comparing the survival curve of cells treated with $10 \mu \mathrm{M}$ AmB supplemented with $200 \mathrm{mM}$ L-NAME to that of cells treated with $10 \mu \mathrm{M} \mathrm{AmB}$, indicating that the rate by which superoxide radicals were formed is 4.21 times faster in the combination treatment, compared to treatment with AmB alone. The same was true for membrane permeabilization events induced by AmB supplemented with L-NAME; upon treatment with the latter, a median survival of $180 \mathrm{~min}$ was observed, as compared to $>180 \mathrm{~min}$ for treatment with AmB alone, and when comparing both survival curves, a hazard ratio of 1.56 was found. This suggested also that membrane permeabilization occurred faster when cells were subjected to AmB in the presence of L-NAME, as compared to treatment with $\mathrm{AmB}$ alone.

We further confirmed that the fast increase in superoxide radical levels during AmB-L-NAME combination treatment was linked to a block in the production of nitric oxide radicals. As we were unable to monitor nitric oxide radicals over time using the DMF platform due to an incompatibility of the DAF-FM DA dye and the DMF setup, we opted to further investigate the kinetics of nitric oxide radical production by flow cytometry. Indeed, upon AmB treatment, yeast cells started to produce nitric oxide radicals from $30 \mathrm{~min}$ onwards $(P<0.0001)$, and a similar trend was observed to that of superoxide radical accumulation during treatment with AmB supplemented with L-NAME (Supplemental Information S2 Figure).

To further elucidate the variation of superoxide radical levels when cells were subjected to $\mathrm{AmB}$ treatment in the presence of L-NAME, we analysed the fluorescence intensity of individual cells. To this end, single cells were monitored over time in $15 \mathrm{~min}$ intervals, and hence the fluorescence intensity of each cell, represented by one dot, was reanalysed every $15 \mathrm{~min}$. We found that the DHE fluorescence intensity of cells during $\mathrm{AmB}$ treatment gradually increased over time, and the highest fluorescence intensity was measured at $180 \mathrm{~min}$, the end point of this study (Figure 3(c)). Compared to the DHE fluorescence at $15 \mathrm{~min}$, the DHE fluorescence intensity was significantly different from $135 \mathrm{~min}$ onwards. In contrast, the DHE fluorescence intensity of cells treated with $10 \mu \mathrm{M}$ AmB supplemented with $200 \mathrm{mM} \mathrm{L}$ NAME showed two subpopulations, suggesting that superoxide radical accumulation took place in a biphasic manner; the first and highest superoxide radical accumulation peak was observed at approximately $75 \mathrm{~min}$, followed by a rather slow decrease and a second peak at approximately $150 \mathrm{~min}$ (Figure 3(d)). Here, the DHE fluorescence intensity was statistically significant from $60 \mathrm{~min}$ onwards (compared to DHE fluorescence at $15 \mathrm{~min}$ ). The kinetics of DHE fluorescence of 35 individual cells, representative for more than 3000 analysed cells, showed different subsets of cells in ROS readouts over time. Some subsets showed an increase in
DHE fluorescence, followed by a decrease in fluorescence at certain time points, while other subsets showed a gradual increase in fluorescence over time (Figure 3(e)). Hence, it seemed that not only the number of cells accumulating superoxide radicals increased when subjected to AmB treatment in the presence of L-NAME, but also the intracellular levels of superoxide radicals were altered in a timedependent manner, as compared to treatment of cells with AmB alone.

Moreover, to further support the data of the single cell analysis via the DMF platform, we performed additional time lapse experiments in bulk via FACS and analysed the subpopulations of DHE- and PI-positive cells of cultures treated with $10 \mu \mathrm{M} \mathrm{AmB}$ in the presence and absence of $200 \mathrm{mM}$ L-NAME for 30, 60, 90, and $180 \mathrm{~min}$. We found an increased number of DHE- and PI-positive cells when yeast was treated with $10 \mu \mathrm{M} A \mathrm{mB}$ supplemented with $200 \mathrm{mM}$ L-NAME as compared to treatment with AmB alone, starting from $30 \mathrm{~min}$ to $90 \mathrm{~min}$ incubation, respectively (Supplemental Information S5 Figure). These bulk data showed faster and increased ROS accumulation and faster membrane permeabilization by $\mathrm{AmB}$ when coincubated with L-NAME and corroborated the data of the single cell analysis via the DMF platform. Note that the number of PI-positive cells induced by $\mathrm{AmB}$ in the presence of $\mathrm{L}$ NAME is higher when assessed in bulk as compared to the DMF setup.

\subsection{Inhibition of Nitric Oxide Radical Accumulation Resulted} in Faster Arrest of Proliferative Capacity of Saccharomyces cerevisiae Cells by Amphotericin B. The results described above indicate that $\mathrm{AmB}$-induced superoxide radical accumulation and membrane permeabilization were significantly altered upon the addition of L-NAME. This tempted us to further investigate whether the proliferative capacity of cells was affected in a time-dependent manner, when comparing both treatments. To this end, plating of S. cerevisiae cultures subjected to both treatments was carried out every $15 \mathrm{~min}$, and the number of cells that were able to proliferate (and form CFU) was determined.

At all time points, the proliferative capacity of cells treated with $10 \mu \mathrm{M} \mathrm{AmB}$ and $200 \mathrm{mM}$ L-NAME was significantly reduced as compared to cells treated with $10 \mu \mathrm{M}$ AmB alone $(P<0.0001)$ (Figure $4(\mathrm{c}))$. In addition, it seemed that the proliferative capacity of cells subjected to AmB-LNAME treatment was reduced very fast, that is, within $15 \mathrm{~min}(P<0.0001)$, whereas the proliferative capacity of cells upon treatment with $\mathrm{AmB}$ alone decreased in a significant manner from 45 min onwards $(P=0.003)$. This suggested that the fast decrease in proliferative capacity of cells within 15 min upon incubation with $\mathrm{AmB}$ and L-NAME was independent of superoxide radical accumulation and membrane permeabilization. Similar observations were made in the survival analyses for superoxide radical accumulation and membrane compromising events (Figures 3(a) and 3(b)) and were supported by a second statistical analysis (Two-way ANOVA followed by Dunnett multiple comparison test). Specifically, a significant difference in the number of cells accumulating superoxide radicals (Figure 4(a)) and 


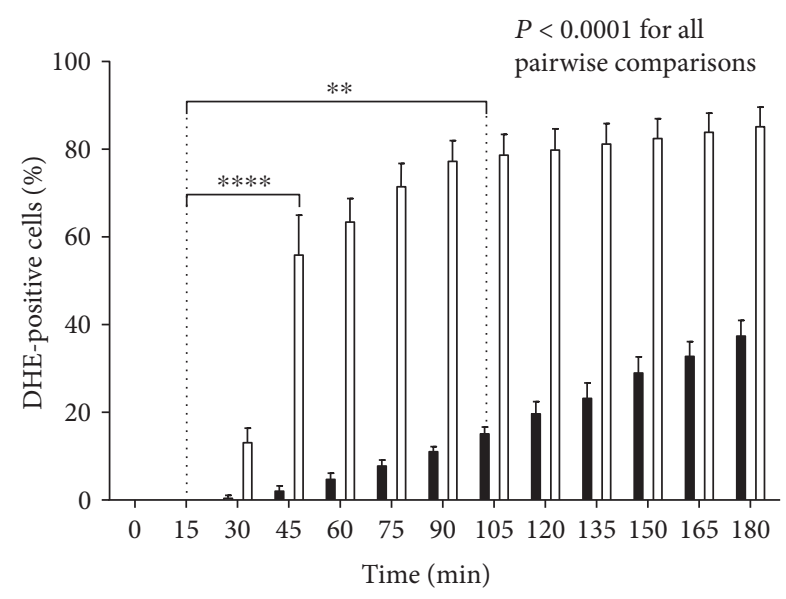

(a)

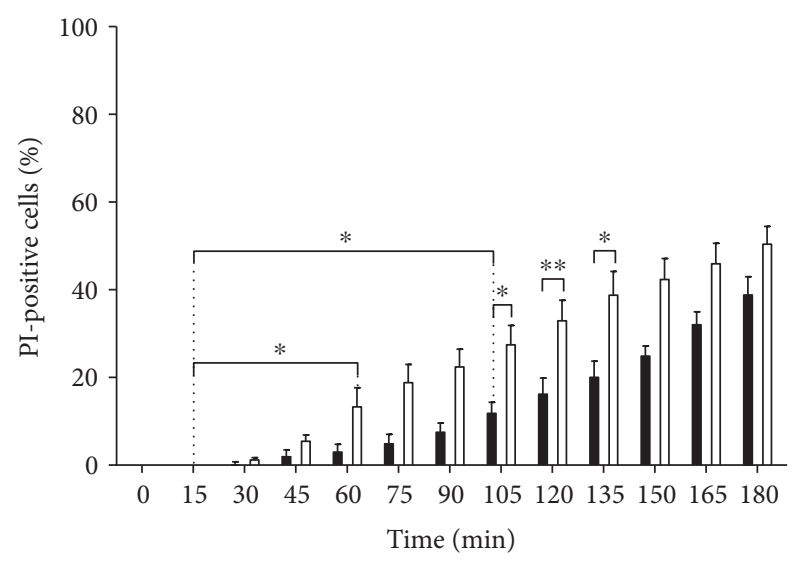

(b)

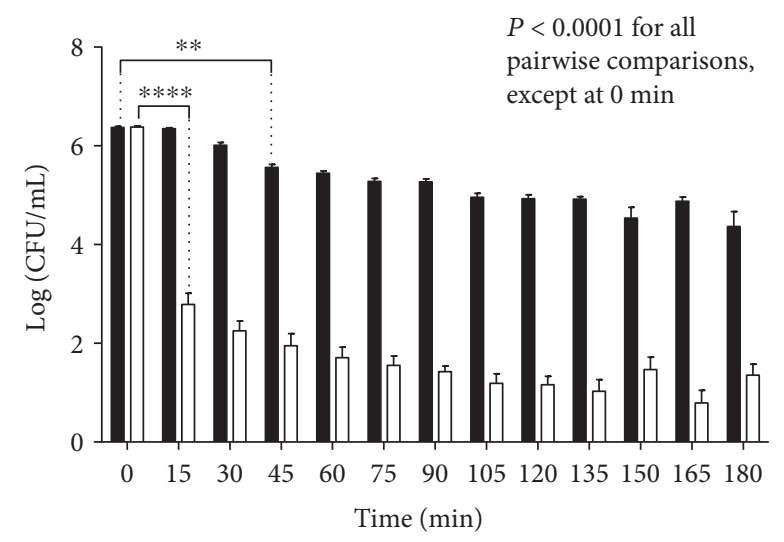

(c)

Figure 4: L-NAME decreased the proliferative capacity of cells during AmB treatment, which seems independent of superoxide radical accumulation. Exponential yeast cells were treated with $10 \mu \mathrm{M}$ AmB in the presence (white bars) or absence (black bars) of $200 \mathrm{mM}$ LNAME for $3 \mathrm{~h}$. Cells were analysed for their DHE and PI fluorescence in the DMF setup (a and b) or subjected to bulk plating assays (c) every $15 \mathrm{~min}$. Means and standard error of the means (SEMs) of at least 3 independent biological experiments $(n \geq 3)$ are presented. Twoway ANOVA followed by Tukey multiple comparison test was performed to analyse significant differences between the two treatments; Two-way ANOVA followed by Dunnett multiple comparison test was performed to analyse significant differences between the first data point (i.e., $0 \mathrm{~min}$ (in (c)) or $15 \mathrm{~min}$ (in (a) and (b))) and other data points within the same treatment (only the primary significant difference is presented to avoid overcrowding of the figure); *,**, and $* * * *$ represent $P<0.05, P<0.01$, and $P<0.0001$, respectively. A dotted line is shown at $15 \mathrm{~min}$ to point out the clear differences between the responses at this time point.

showing membrane permeabilization (Figure 4(b)) was found at earlier time points (i.e., $45 \mathrm{~min}$ versus $105 \mathrm{~min}$ for superoxide radical accumulation and $60 \mathrm{~min}$ versus $105 \mathrm{~min}$ for membrane permeabilization) when cells were treated with AmB in the presence of L-NAME, as compared to cells treated with AmB alone.

However, although approximately $99.5 \%$ of the treated population was not able to proliferate from 15 min onwards (Figure 4(c)) when subjected to AmB-L-NAME treatment, they were still able to accumulate superoxide radicals at that point, which resulted in a superoxide radical boost starting at $30 \mathrm{~min}$ (Figure 4(a)). Hence, it seemed that these cells were still metabolically active and possibly used increased intracellular superoxide radical levels to enter a programmed cell death pathway. In contrast, loss of proliferative capacity of cells treated with AmB alone (Figure 4(c)) might be explained by the gradual increase in the number of membrane permeabilization events; a similar trend in both curves was observed (Figure 4(b)).

To further confirm the crucial role of nitric oxide in modulating AmB's fungicidal activity, we performed checkerboard assays with $\mathrm{AmB}$ and the nitric oxide donor, S-nitrosoglutathione. We found that $2 \mathrm{mM} \mathrm{S}$ nitrosoglutathione reduced the activity of AmB (Figure 5). Hence, nitric oxide plays an important role in modulating AmB's activity.

3.5. Amphotericin B Induced Cell Cycle Arrest in Saccharomyces cerevisiae Independently of L-NAME. We further analysed whether the increased loss of proliferative capacity upon combined AmB and L-NAME treatment within 15 min of treatment can be attributed to cell cycle arrest. To this end, we analysed the fraction of cells in the G0/G1, S, and G2/M cell cycle phases at the beginning and 


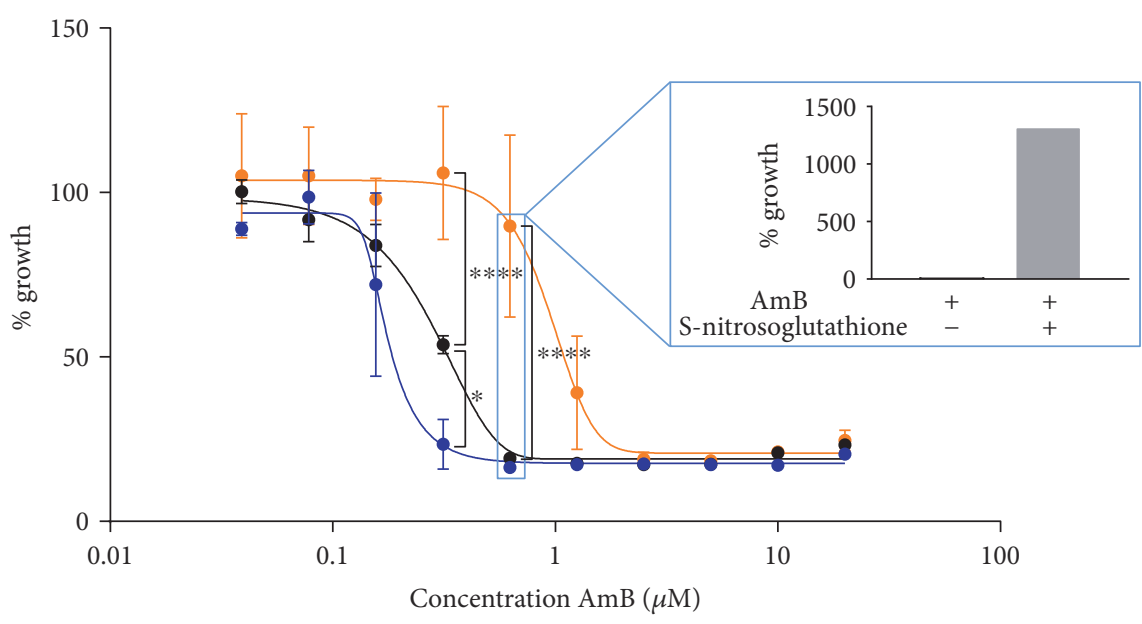

FIgure 5: The nitric oxide donor, S-nitrosoglutathione, inhibited the killing activity of AmB. Yeast cells were treated with different concentrations of AmB, in the absence (black) or presence of $200 \mathrm{mM}$ L-NAME (blue) or $2 \mathrm{mM}$ S-nitrosoglutathione (orange). Means and standard errors of the means (SEMs) of at least two independent biological experiments $(n \geq 2)$ are presented. The number of CFU/mL for different treatments (insert) was assessed by plating assays and CFU counting and is shown relative to the number of CFU/mL at the start of the experiment. Two-way ANOVA followed by Tukey multiple comparison test was performed to analyse significant differences between the two treatments; $*$ and $* * * *$ represent $P<0.05$ and $P<0.0001$, respectively.

after 7.5 and 15 min of incubation with either $\mathrm{AmB}$ alone or AmB supplemented with L-NAME.

$\mathrm{AmB}$ induced cell cycle arrest in the G2/M phase in yeast at $15 \mathrm{~min}$ of treatment, as compared to control cells $(P<0.0001)$ (Figure 6). Interestingly, treatment with $\mathrm{L}$ NAME alone resulted in a decrease in the amount of cells in the $S$ phase at 7.5 and $15 \mathrm{~min}(P=0.03$ and $P=0.0009$, resp.) and an increase in the amount of cells in the G2/M phase at $15 \mathrm{~min}(P=0.03)$, compared to control-treated cells. Yet, L-NAME alone did not increase the other cell cycle phase distributions in a significant manner at both time points, compared to control-treated cells $(P>0.05$ at $7.5 \mathrm{~min}$ and $15 \mathrm{~min}$ of treatment). Treatment with AmB-LNAME did not significantly alter cell cycle phase distributions as compared to those in treatment with $\mathrm{AmB}$ alone, suggesting that the observed increased loss of proliferative capacity of yeast cells treated with the AmB-L-NAME combination, compared to $\mathrm{AmB}$ treatment alone, was not due to increased cell cycle arrest.

3.6. Candida albicans and Candida glabrata Were More Susceptible to Amphotericin B Treatment in the Presence of Nitric Oxide Radical Production Inhibitors. To validate the results obtained in yeast and in support of the clinical relevance of $\mathrm{AmB}$ treatment in the presence of L-NAME, we investigated the effects of this treatment on the human pathogen Candida albicans. We confirmed that AmB induced superoxide and nitric oxide radical accumulation $(P<0.0001$ at $10 \mu \mathrm{M} \mathrm{AmB})$, associated with loss of proliferative capacity, in $C$. albicans, in a similar dose-dependent way as was observed for S. cerevisiae (Figure 7). These results indicated that the range of $\mathrm{AmB}$ concentrations used for $S$. cerevisiae was applicable for $C$. albicans as well. Furthermore, we assessed whether treatment at $37^{\circ} \mathrm{C}$ with $\mathrm{AmB}$ in the presence of L-NAME also significantly affected the number of cells that are able to proliferate as compared to treatment with $\mathrm{AmB}$ alone. At $37^{\circ} \mathrm{C}$, we found that $5 \mu \mathrm{M} \mathrm{AmB}$ resulted in killing of the C. albicans culture by 2 Log units (99.00\%) and $10 \mu \mathrm{M}$ AmB by 4 Log units (99.99\%). These values are in line with the reported minimal fungicidal concentration (MFC) of $\mathrm{AmB}(8.66 \mu \mathrm{M})$ in a similar experimental setup [48]. Coincubation of $200 \mathrm{mM} \mathrm{L-}$ NAME and $5 \mu \mathrm{M}$ AmB significantly reduced the number of CFUs as compared to treatment with AmB alone $(P<0.05)$ (Figure 8(a)), indicating that L-NAME also enhanced AmB's fungicidal activity against $C$. albicans. Also, in case of C. glabrata, we found that $10 \mu \mathrm{M} A \mathrm{mB}$ resulted in killing of C. glabrata by 4 Log units (99.99\%). These values are in line with the reported MFC of AmB against C. glabrata $(17 \mu \mathrm{M})$ [49]. Also here, $200 \mathrm{mM}$ L-NAME significantly increased AmB's fungicidal activity against $C$. glabrata (Figure 8(b)). All these data point to the clinical potential of combining AmB with L-NAME.

\section{Discussion}

The aim of this study was to investigate and understand how AmB-induced oxidative and nitrosative stresses (characterized by excess of superoxide radicals and nitric oxide radicals, resp.) are linked to fungal cell death. To inhibit the generation of nitric oxide radicals and nitrosative stress in cells, we used the nitric oxide synthase inhibitor LNAME. From the bulk studies, we found that superoxide radical accumulation increased when nitric oxide production was inhibited, thereby increasing AmB's antifungal activity. We then further assessed the kinetics of superoxide radical accumulation, membrane permeabilization, and loss of proliferative capacity using a DMF platform in which individual $S$. cerevisiae cells were captured and monitored for their responses over time during treatment. As seeding of C. albicans was problematic due to the presence of hyphae, we first tested our hypotheses on $S$. cerevisiae and translated the most 


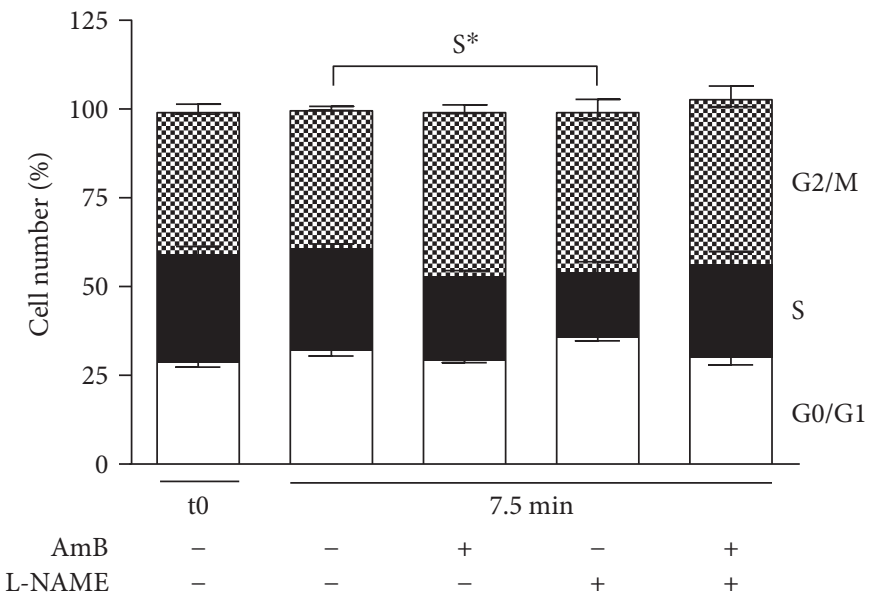

(a)

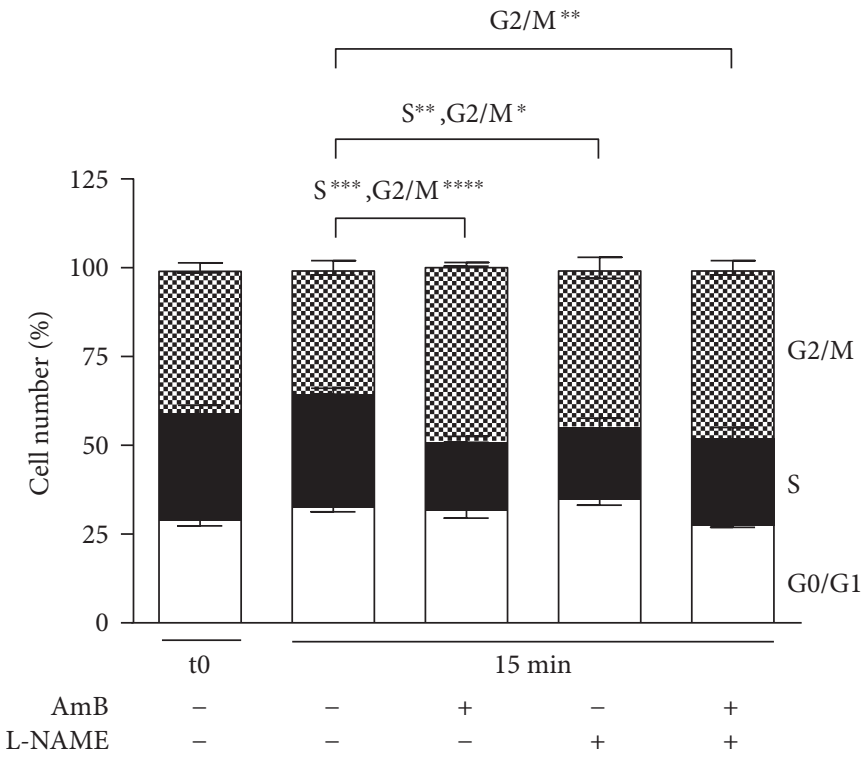

(b)

FIGURE 6: Amphotericin B induced cell cycle arrest in the G2/M phase in yeast. Exponential yeast cultures were treated with either control (1\% DMSO; 10\% mQ), $200 \mathrm{mM} \mathrm{L-NAME} \mathrm{(dissolved} \mathrm{in} \mathrm{mQ),} 10 \mu \mathrm{M} \mathrm{AmB}$ (dissolved in DMSO), or a combination of the above for $7.5 \mathrm{~min}(\mathrm{a})$ and 15 min (b). After treatment, cells were washed with PBS, fixed in $70 \% \mathrm{EtOH}$, stained with PI, and subjected to flow cytometry for cell cycle analysis. White bars represent cells in the G0/G1 phase, black bars represent cells in the S phase, and pixelated bars represent cells in the G2/M phase. Means and standard error of the means (SEMs) of 3 independent biological experiments $(n=3)$ are presented. Two-way ANOVA followed by Tukey multiple comparison test was performed to analyse differences between the cell cycle distributions of control treatment and AmB, L-NAME, or AmB $+\mathrm{L}-\mathrm{NAME}$ treatment and between cell cycle distributions of AmB treatment and AmB $+\mathrm{L}-\mathrm{NAME}$ treatment. $*, * *, * * *$, and $* * * *$ represent $P<0.05, P<0.01, P<0.001$, and $P<0.0001$, respectively. Multiplicity-adjusted $P$ values are presented in the main text.

prominent findings to C. albicans and C. glabrata afterwards using bulk assays. We showed that L-NAME increased and accelerated the effect of AmB on the accumulation of superoxide radicals, membrane permeabilization, and loss of proliferative capacity in S. cerevisiae. Moreover, we showed that the data obtained via time lapse experiments in bulk corroborates the data of the single-cell analysis via the DMF platform (Supplemental Information S5 Figure). We revealed that superoxide radicals are important mediators for AmBinduced fungal cell death. However, L-NAME could only increase the killing potential of $\mathrm{AmB}$, but not that of peroxide. This implies an AmB-specific effect of L-NAME and might point to L-NAME's effects via an ergosteroldependent pathway. Indeed, ROS generation by AmB has been described as a consequence of AmB's spontaneous insertion into ergosterol-containing membranes [50, 51]. In contrast, nitric oxide radicals seemed to play a role in mediating tolerance towards $\mathrm{AmB}$, pointing to a beneficial role of nitric oxide radicals in the yeast response towards AmB. We found that cellular responses are classified into two groups based on the time point that they occur, that is, within $15 \mathrm{~min}$ and from 30 to $45 \mathrm{~min}$ onwards (Figure 9). 


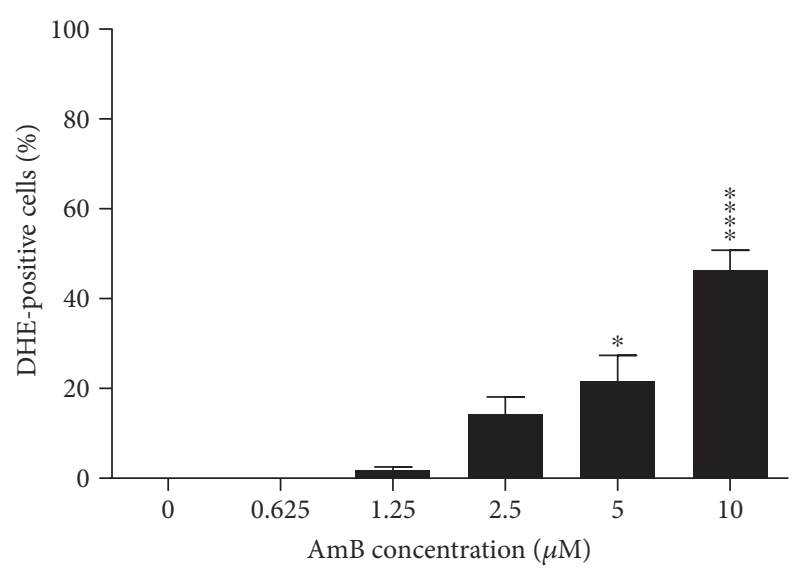

(a)

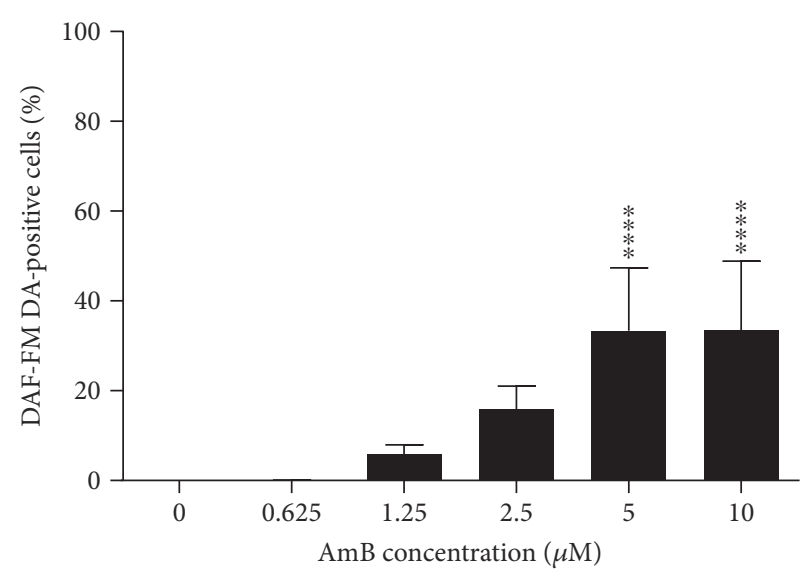

(b)

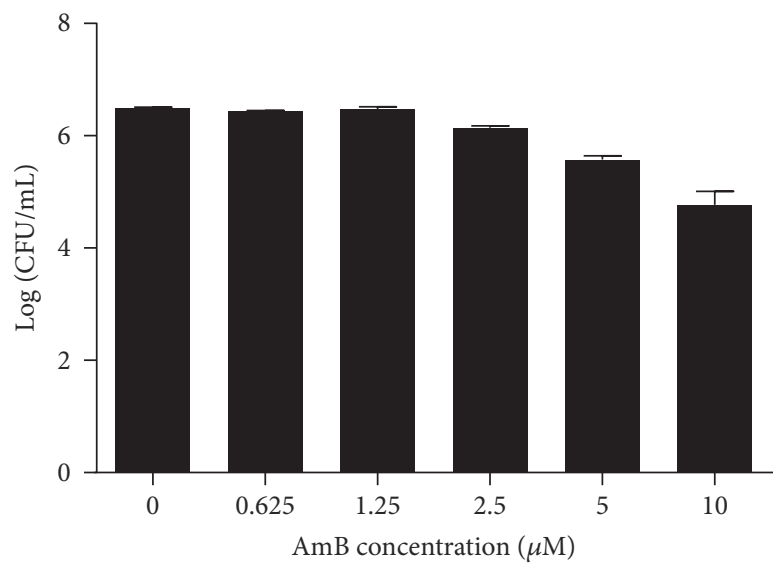

(c)

Figure 7: AmB induced accumulation of superoxide and nitric oxide radicals in C. albicans and decreased the number of cells that are able to proliferate. Exponential C. albicans cultures were treated with different concentrations of AmB for $3 \mathrm{~h}$ at room temperature and subjected to flow cytometry or plating assays. (a) Levels of superoxide radical detected by dihydroethidium (DHE) fluorescence and flow cytometry. (b) Levels of nitric oxide radical detected by 4 -amino-5-methylamino-2', $7^{\prime}$-difluorofluorescein diacetate (DAF-FM DA) fluorescence and flow cytometry. (c) Number of CFU/mL in Log-scale, assessed by plating assays and CFU counting. Means and standard errors of the means (SEMs) of at least 3 independent biological experiments $(n \geq 3)$ are presented. Two-way ANOVA followed by Dunnett multiple comparison test was performed to analyse statistically significant differences in the number of DHE- and DAF-FM DA-positive cells and cells able to proliferate between control treatment and treatment with different concentrations of AmB. $*$ and $* * * *$ represent $P<0.05$ and $P<0.0001$, respectively.

Upon treatment of S. cerevisiae with AmB in the presence of L-NAME, not only an increased level of superoxide radicals was found as compared to treatment with AmB alone, but also an accelerating effect on these levels was observed (Figures 3(a) and 3(b)). Our DMF approach, allowing a detailed kinetic study at a single-cell level, showed that superoxide radicals accumulated in a biphasic manner during $\mathrm{AmB}$ treatment in the presence of L-NAME, resulting in two superoxide radical accumulation peaks at $75 \mathrm{~min}$ and $150 \mathrm{~min}$, respectively. This was not observed for cells treated with AmB in the absence of L-NAME, where a superoxide radical accumulation peak seemed to manifest at $180 \mathrm{~min}$, the endpoint of this study (Figures 3(c) and 3(d)). Interestingly, all cell responses, being superoxide radical accumulation, membrane permeabilization, and loss of proliferative capacity, presented themselves significantly faster, as compared to these responses during treatment with $\mathrm{AmB}$ alone. Therefore, it might well be that L-NAME solely accelerated AmB action with respect to superoxide radical accumulation and membrane permeabilization, and hence, similar outcomes might be expected for treatment with AmB alone over a longer period of time (i.e., >180 min). Whether, this is the case that requires further investigation. This hypothesis is supported by the fact that L-NAME acts fungistatic (MIC against S. cerevisiae of $250 \mathrm{mM}$ ), however not fungicidal when administered alone (Supplemental Information S6 Figure), and does not affect the level of superoxide radicals, membrane permeabilization, and proliferative capacity of control cells (Figure 2), suggesting that the observed effect on cell responses is not caused by a similar and dual action of L-NAME and AmB, as is often the case for synergistic interactions.

Secondly, and most notably, L-NAME had a strong enhancing effect on AmB-induced loss of proliferative 


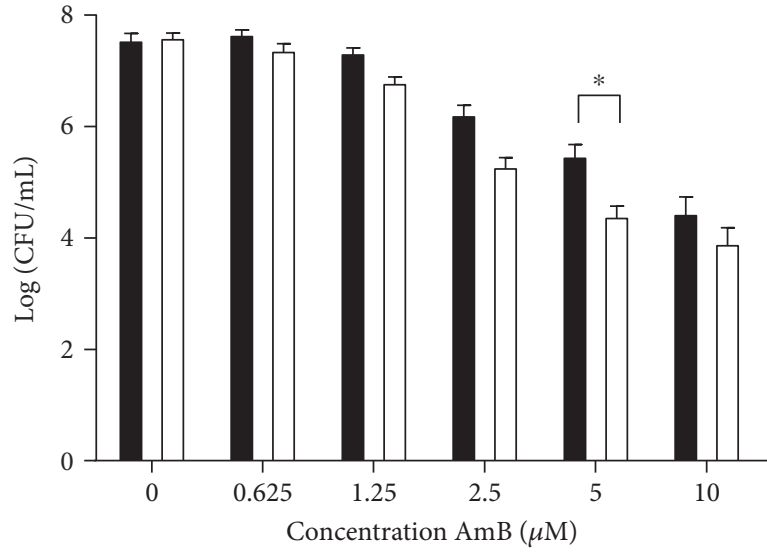

(a)

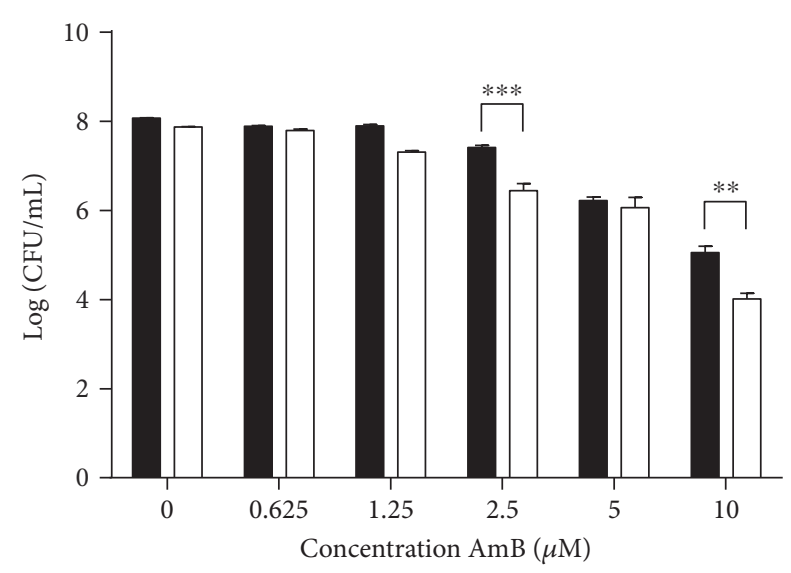

(b)

Figure 8: L-NAME significantly decreased the number of AmB-treated cells that are able to proliferate in C. albicans (a) and C. glabrata (b). Exponential C. albicans and C. glabrata cultures were treated with different dosages of AmB in the presence (white bars) or absence (black bars) of $200 \mathrm{mM} \mathrm{L-NAME}$ for $180 \mathrm{~min}$ at $37^{\circ} \mathrm{C}$ and subjected to plating assays. Means and standard errors of the means (SEMs) of 3 independent biological experiments $(n=3)$ are presented. Two-way ANOVA followed by Tukey multiple comparison test was performed to analyse significant differences between the two treatments; $*, * *$, and $* * *$ represent $P<0.05, P<0.01$, and $P<0.001$, respectively.

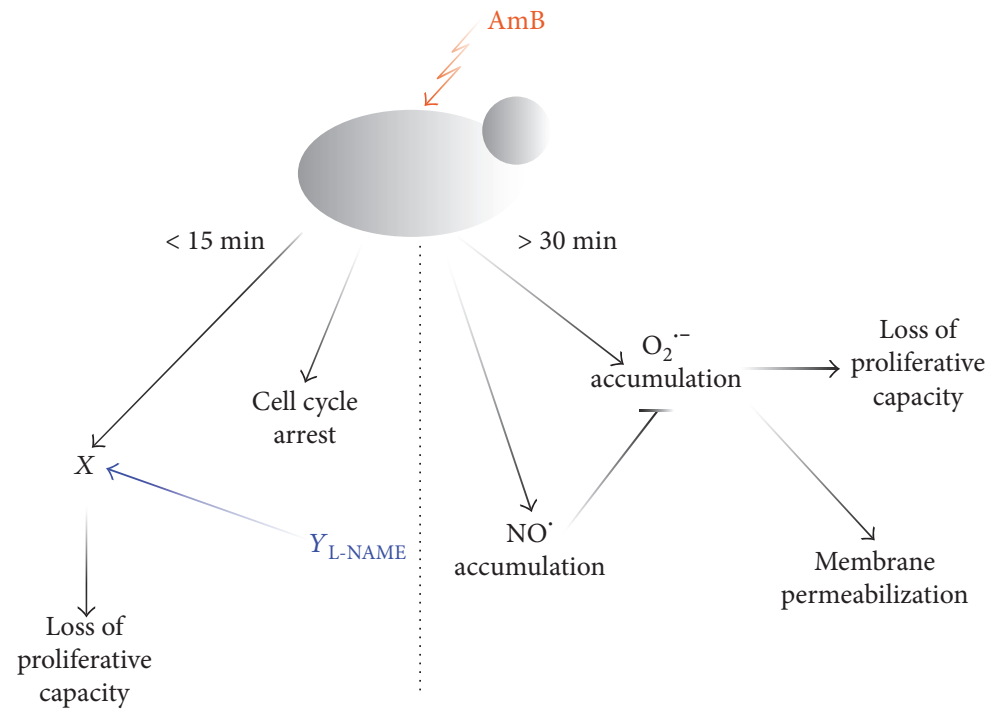

Figure 9: Schematic overview of the major findings on AmB mechanism of action in this study. Within $15 \mathrm{~min}$, AmB caused cell cycle arrest in the G2/M phase and induced a yet to be elucidated event $X$, the latter leading to loss of proliferative capacity in yeast. These effects were independent of nitric oxide radicals, superoxide anion radicals, and membrane permeabilization. After $30 \mathrm{~min}$, AmB induced the accumulation of superoxide radicals, which was associated with membrane permeabilization and loss of proliferative capacity in yeast, and was partially blocked by beneficial action of nitric oxide radicals. Interestingly, the combinatorial action of AmB and L-NAME induced a yet to be identified event $Y$ within $15 \mathrm{~min}$, which was independent of nitric oxide radicals, and enhanced the effect of event $X$, leading to enhanced loss of proliferative capacity in yeast.

capacity in yeast: within $15 \mathrm{~min}$, approximately $99.5 \%$ of the cells lost their proliferative capacity when subjected to AmB treatment in the presence of L-NAME. In contrast, treatment with $\mathrm{AmB}$ alone did not reach a similar negative impact on proliferative capacity of cells within $180 \mathrm{~min}$ (Figure 4(c)). This suggest also that here nitric oxide radicals play an important, beneficial, role in the response towards AmB. Yet, we showed that nitric oxide radicals only accumulated from 30 min onwards (Supplemental Information S2 Figure). Hence, L-NAME seemed to have an additional effect apart from inhibiting nitric oxide radical production, resulting in enhancement of AmB fungicidal activity, and this effect occurred within 15 min of treatment (event $Y$ in Figure 9). Interestingly, cells receiving treatment with $\mathrm{AmB}$ and $\mathrm{L}$ NAME were able to accumulate superoxide radicals only after $30 \mathrm{~min}$, suggesting that these accumulated positive cells were still metabolically active and that AmB-L-NAMEtreated cells might use increased levels of superoxide radicals, and thus oxidative stress, to enter a programmed cell death pathway. In addition, as the rapid loss of proliferative 
capacity of cells upon combined treatment with AmB and L-NAME could not be explained by superoxide radical accumulation and membrane permeabilization, it seems that mechanisms other than these underlie the negative effect on the proliferative capacity of cells during the first $15 \mathrm{~min}$ of $\mathrm{AmB}$ treatment in the presence of L-NAME (i.e., event $\mathrm{X}$ in Figure 9). A plausible explanation for the loss of proliferative capacity, independent of oxidative stress and nonapoptotic cell death, is cell cycle arrest. We showed that $\mathrm{AmB}$ induced cell cycle arrest in the G2/M phase in yeast within $15 \mathrm{~min}$ of treatment. These data are in line with other reports on the effect of $\mathrm{AmB}$ on the cell cycle in mammalian cell lines [52, 53]. However, this effect was found to be independent of L-NAME, indicating that cell cycle arrest could not account for the observed increased loss of proliferative capacity when cells were treated with the AmB-L-NAME combination.

Overall, it seems that nitric oxide radicals play a beneficial role in AmB antifungal activity, as further demonstrated by the S-nitrosoglutathione-induced inhibition of AmB's killing activity (Figure 5). Nitric oxide radicals were previously shown to protect bacteria against a wide spectrum of antibiotics by alleviating the oxidative stress imposed by them [54]. In addition, nitric oxide radicals were reported to affect fungal cell death, both in beneficial and destructive manners. Specifically, increased intracellular nitric oxide radical levels are suggested to play a cytoprotective role in yeast during stress from heat-shock and hydrostatic pressure [55]. In contrast, PAF26-induced production of nitric oxide radicals was correlated to its antifungal activity, and administering L-NAME partially restored yeast growth in the presence of PAF26, indicating that nitric oxide radicals play an important role in PAF26-induced cell death [44]. In line, Almeida and colleagues showed that nitric oxide is a crucial mediator of $\mathrm{H}_{2} \mathrm{O}_{2}$-induced apoptosis in yeast and that blockage of nitric oxide radical production by L-NAME decreased the intracellular levels of ROS, thereby increasing survival [46]. Interestingly, in our study, L-NAME increased the accumulation of superoxide radicals during AmB treatment, while decreasing the proliferative capacity of cells in the presence of $\mathrm{AmB}$, and thus decreasing survival. It seems that a nitric oxide radical-dependent tolerance system is switched on upon AmB treatment in yeast, perhaps similar to the system recently described by Nasuno and colleagues [56]. In that study, a downstream pathway of nitric oxide radicals involved in high-temperature stress tolerance in yeast was unravelled. They showed that nitric oxide radicals activated the transcription factor Macl that on its turn induced the CTR1 gene and resulted in increased cellular copper levels, which then resulted in activation of Sod1, a superoxide dismutase [56]. Alternatively, it could also be that nitric oxide activates, potentially via S-nitrosylation, $\mathrm{AmB}$ tolerance pathways such as the yeast HOG pathway $[57,58]$. How exactly tolerance to $\mathrm{AmB}$ via nitric oxide production is mediated requires further investigation.

We further translated the most prominent findings to the human pathogens, Candida albicans and Candida glabrata, and found that treatment of C. albicans or C. glabrata with $\mathrm{AmB}$ in the presence of L-NAME significantly increases the loss of proliferative capacity, as compared to treatment with $\mathrm{AmB}$ alone, suggesting that treatment of AmB in the presence of L-NAME might have a clinical relevance. L-NAME has been extensively studied in in vitro, ex vivo, and in vivo systems (reviewed in [59]). It was shown to inhibit corneal angiogenesis under chemical growth factor stimulation in rabbits [60] and improve leucocyte adherence and emigration to venular endothelium, characteristic of acute inflammation, in cat jejuni [61]. In addition, L-NAME was found to modulate hemodynamics in dogs [62], ewes [63], and guinea pigs [64] and was shown to reverse sepsis-associated hypotension in various animal models [65]. In humans, LNAME was tested to treat hypotension, asthma, and sepsis. In view of the latter, L-NAME increased the systemic vascular resistance and blood pressure in septic patients $[66,67]$. In treatment of asthma, no adverse effects were found in healthy volunteers and patients with asthma, and results on exhaled nitric oxide levels indicated that L-NAME might be used for treatment of asthma [68]. Finally, L-NAME increased the mean arterial pressure and cerebral blood flow, treating hypotension in patients with tetraplegia. No adverse effects on healthy volunteers or patients were found [69-71]. Hence, although L-NAME as such is not used in a clinical setting to date, it has been studied extensively in humans during the past decades.

$\mathrm{AmB}$, on the other hand, is used in clinical settings to treat invasive fungal infections. However, its applicability is limited due to its nephrotoxicity and hence, it must be used with care [26]. Recent findings indicated that AmB exerts its antifungal action by extracting ergosterol from the plasma membrane, resulting in loss of cell membrane integrity, interference with ergosterol-depending cellular processes, and ultimately cell death [29]. In addition, AmB treatment causes a significant loss of fungal replication competency and numerous morphological and physiological effects on susceptible yeast cells, including cytoplasm shrinking, abnormal nuclear and mitochondrial morphologies, and oxidative stress [72]. Finally, Teixeira-Santos and colleagues showed that pathogenic and nonpathogenic yeast cells develop compensatory responses towards $\mathrm{AmB}$ treatment, related to membrane polarization, metabolic activity, and ROS production, depending on the drug concentration and the duration of the treatment [14]. Likewise, we found that treatment of yeast cells with clinically relevant $\mathrm{AmB}$ concentrations (i.e., $0.1 \mu \mathrm{M}$ to $21.6 \mu \mathrm{M} \mathrm{AmB}[14,73])$ induces the accumulation of superoxide radicals, in addition to nitric oxide radicals, in $S$. cerevisiae and C. albicans. Furthermore, we found that clinically relevant AmB concentrations significantly increase the loss of proliferative capacity of $S$. cerevisiae, C. albicans, and C. glabrata in the presence of L-NAME.

\section{Conclusions}

In conclusion, we showed that L-NAME can increase and accelerate AmB-induced superoxide radical accumulation and loss of proliferative capacity in S. cerevisiae, the latter was confirmed in the human pathogens C. albicans and $C$. glabrata. Moreover, we found that the production of nitric oxide radicals seems to constitute a tolerance mechanism 
that is induced by $\mathrm{AmB}$ treatment and partially counteracts $\mathrm{AmB}$ activity. Moreover, the combinatorial action of AmB and L-NAME induced an additional, yet to be elucidated, event that further enhanced AmB's fungicidal activity. The effects of both AmB and L-NAME have been extensively studied in various in vitro and in vivo models, pointing towards the potential of AmB-L-NAME combination treatment. However, further research on pharmacology and toxicology of the AmB-L-NAME combination needs to be performed in order to assess its potential clinical relevance.

\section{Conflicts of Interest}

The authors declare that there is no conflict of interest regarding the publication of this article.

\section{Authors' Contributions}

Kim Vriens, Caroline Struyfs, and Tanne L. Cools performed all cytotoxicity assays in bulk; Phalguni Tewari Kumar performed all cytotoxicities on the DMF platform; Belém Sampaio-Marques carried out cell cycle analysis; Kim Vriens, Phalguni Tewari Kumar, and Caroline Struyfs performed data analysis, and Kim Vriens, Phalguni Tewari Kumar, Caroline Struyfs, and Pieter Spincemaille wrote the manuscript. Tadej Kokalj, Paula Ludovico, Jeroen Lammertyn, Bruno P. A. Cammue, and Karin Thevissen supervised the study. All authors critically read and approved the manuscript. Kim Vriens, Phalguni Tewari Kumar, and Caroline Struyfs contributed equally to this work and hence are the shared first authors.

\section{Acknowledgments}

Kim Vriens acknowledges the receipt of a predoctoral grant from the Flanders Innovation \& Entrepeneurship Agency (IWT-SB 111016); Karin Thevissen acknowledges the receipt of a mandate of Industrial Research Fund (KU Leuven). In addition, the research leading to these results has received funding from the Research Foundation - Flanders (FWO G086114N and G080016N) and the KU Leuven (OT 13/ 058 and IDO 10/012, IOF KP/12/009 Atheromix, IOF KP/ $12 / 002$ Nanodiag). This work was partially developed under the scope of the project NORTE-01-0145-FEDER-000013, supported by the Northern Portugal Regional Operational Programme (NORTE 2020), under the Portugal 2020 Partnership Agreement, through the European Regional Development Fund (FEDER). Belém Sampaio-Marques is supported by the fellowship SFRH/BPD/90533/2012 funded by Fundação para a Ciência e Tecnologia (FCT, Portugal).

\section{References}

[1] A. J. Brown, K. Haynes, and J. Quinn, "Nitrosative and oxidative stress responses in fungal pathogenicity," Current Opinion in Microbiology, vol. 12, no. 4, pp. 384-391, 2009.

[2] D. Kaloriti, A. Tillmann, E. Cook et al., "Combinatorial stresses kill pathogenic Candida species," Medical Mycology, vol. 50, no. 7, pp. 699-709, 2012.
[3] C. K. Ferrari, P. C. Souto, E. L. Franca, and A. C. HonorioFranca, "Oxidative and nitrosative stress on phagocytes' function: from effective defense to immunity evasion mechanisms," Archivum Immunologiae et Therapiae Experimentalis, vol. 59, no. 6, pp. 441-448, 2011.

[4] B. S. Hromatka, S. M. Noble, and A. D. Johnson, "Transcriptional response of Candida albicans to nitric oxide and the role of the YHB1 gene in nitrosative stress and virulence," Molecular Biology of the Cell, vol. 16, no. 10, pp. 4814-4826, 2005.

[5] P. Moradas-Ferreira and V. Costa, "Adaptive response of the yeast Saccharomyces cerevisiae to reactive oxygen species: defences, damage and death," Redox Report, vol. 5, no. 5, pp. 277-285, 2000.

[6] N. Chauhan, J. P. Latge, and R. Calderone, "Signalling and oxidant adaptation in Candida albicans and Aspergillus fumigatus," Nature Reviews Microbiology, vol. 4, no. 6, pp. 435-444, 2006.

[7] M. Cuellar-Cruz, E. Lopez-Romero, E. Ruiz-Baca, and R. Zazueta-Sandoval, "Differential response of Candida albicans and Candida glabrata to oxidative and nitrosative stresses," Current Microbiology, vol. 69, no. 5, pp. 733-739, 2014.

[8] N. Delattin, B. P. A. Cammue, and K. Thevissen, "Reactive oxygen species-inducing antifungal agents and their activity against fungal biofilms," Future Medicinal Chemistry, vol. 6, no. 1, pp. 77-90, 2014.

[9] A. Bink, D. Vandenbosch, T. Coenye, H. Nelis, B. P. Cammue, and K. Thevissen, "Superoxide dismutases are involved in Candida albicans biofilm persistence against miconazole," Antimicrobial Agents and Chemotherapy, vol. 55, no. 9, pp. 4033-4037, 2011.

[10] D. Vandenbosch, K. Braeckmans, H. J. Nelis, and T. Coenye, "Fungicidal activity of miconazole against Candida spp. biofilms," The Journal of Antimicrobial Chemotherapy, vol. 65, no. 4, pp. 694-700, 2010.

[11] C. D. Mahl, C. S. Behling, F. S. Hackenhaar et al., "Induction of ROS generation by fluconazole in Candida glabrata: activation of antioxidant enzymes and oxidative DNA damage," Diagnostic Microbiology and Infectious Disease, vol. 82, no. 3, pp. 203208, 2015.

[12] C. E. Linares, S. R. Giacomelli, D. Altenhofen, S. H. Alves, V. M. Morsch, and M. R. Schetinger, "Fluconazole and amphotericin-B resistance are associated with increased catalase and superoxide dismutase activity in Candida albicans and Candida dubliniensis," Revista da Sociedade Brasileira de Medicina Tropical, vol. 46, no. 6, pp. 752-758, 2013.

[13] M. Blatzer, E. Jukic, W. Posch et al., "Amphotericin B resistance in Aspergillus terreus is overpowered by co-application of pro-oxidants," Antioxidants \& Redox Signaling, vol. 23, no. 18, pp. 1424-1438, 2015.

[14] R. Teixeira-Santos, E. Ricardo, S. G. Guerreiro, S. Costa-deOliveira, A. G. Rodrigues, and C. Pina-Vaz, "New insights regarding yeast survival following exposure to liposomal amphotericin B," Antimicrobial Agents and Chemotherapy, vol. 59, no. 10, pp. 6181-6187, 2015.

[15] A. C. Mesa-Arango, N. Trevijano-Contador, E. Román et al., "The production of reactive oxygen species is a universal action mechanism of Amphotericin B against pathogenic yeasts and contributes to the fungicidal effect of this drug," Antimicrobial Agents and Chemotherapy, vol. 58, no. 11, pp. 6627-6638, 2014.

[16] F. Sangalli-Leite, L. Scorzoni, A. C. Mesa-Arango et al., "Amphotericin B mediates killing in Cryptococcus neoformans through the induction of a strong oxidative burst," Microbes and Infection, vol. 13, no. 5, pp. 457-467, 2011. 
[17] J. Kelly, R. Rowan, M. McCann, and K. Kavanagh, "Exposure to caspofungin activates cap and hog pathways in Candida albicans," Medical Mycology, vol. 47, no. 7, pp. 697-706, 2009.

[18] K. Wang, W. Dang, J. Xie et al., "Antimicrobial peptide protonectin disturbs the membrane integrity and induces ROS production in yeast cells," Biochimica et Biophysica Acta (BBA) - Biomembranes, vol. 1848, no. 10, Part A, p. 21, 2015.

[19] T. Wang, G. Shi, J. Shao et al., "In vitro antifungal activity of baicalin against Candida albicans biofilms via apoptotic induction," Microbial Pathogenesis, vol. 87, pp. 21-29, 2015.

[20] K. Vriens, B. P. Cammue, and K. Thevissen, "Antifungal plant defensins: mechanisms of action and production," Molecules, vol. 19, no. 8, pp. 12280-12303, 2014.

[21] A. M. Aerts, L. Bammens, G. Govaert et al., "The antifungal plant Defensin HsAFP1 from Heuchera sanguinea induces apoptosis in Candida albicans," Frontiers in Microbiology, vol. 2, p. 47, 2011.

[22] A. M. Aerts, I. E. François, E. M. Meert, Q. T. Li, B. P. Cammue, and K. Thevissen, "The antifungal activity of RsAFP2, a plant defensin from raphanus sativus, involves the induction of reactive oxygen species in Candida albicans," Journal of Molecular Microbiology and Biotechnology, vol. 13, no. 4, pp. 243-247, 2007.

[23] B. M. E. Hayes, M. R. Bleackley, J. L. Wiltshire, M. A. Anderson, A. Traven, and N. L. van der Weerden, "Identification and mechanism of action of the plant Defensin NaD1 as a new member of the antifungal drug arsenal against Candida albicans," Antimicrobial Agents and Chemotherapy, vol. 57, no. 8, pp. 3667-3675, 2013.

[24] G. F. Ferreira, L. de Matos Baltazar, J. R. Santos et al., “The role of oxidative and nitrosative bursts caused by azoles and amphotericin B against the fungal pathogen Cryptococcus gattii," The Journal of Antimicrobial Chemotherapy, vol. 68, no. 8, pp. 1801-1811, 2013.

[25] E. O. Mello, S. F. Ribeiro, A. O. Carvalho et al., "Antifungal activity of $P v \mathrm{D} 1$ defensin involves plasma membrane permeabilization, inhibition of medium acidification, and induction of ROS in fungi cells," Current Microbiology, vol. 62, no. 4, pp. 1209-1217, 2011.

[26] A. Lemke, A. F. Kiderlen, and O. Kayser, "Amphotericin B," Applied Microbiology and Biotechnology, vol. 68, no. 2, pp. 151-162, 2005.

[27] A. J. Phillips, I. Sudbery, and M. Ramsdale, "Apoptosis induced by environmental stresses and amphotericin B in Candida albicans," Proceedings of the National Academy of Sciences of the United States of America, vol. 100, no. 24, pp. 14327-14332, 2003.

[28] R. S. Al-Dhaheri and L. J. Douglas, "Apoptosis in Candida biofilms exposed to amphotericin B," Journal of Medical Microbiology, vol. 59, Part 2, pp. 149-157, 2010.

[29] T. M. Anderson, M. C. Clay, A. G. Cioffi et al., "Amphotericin forms an extramembranous and fungicidal sterol sponge," Nature Chemical Biology, vol. 10, no. 5, pp. 400406, 2014.

[30] P. T. Kumar, K. Vriens, M. Cornaglia et al., "Digital microfluidics for time-resolved cytotoxicity studies on single non-adherent yeast cells," Lab on a Chip, vol. 15, no. 8, pp. 1852-1860, 2015.

[31] M. E. Cardenas, M. C. Cruz, M. Del Poeta, N. Chung, J. R. Perfect, and J. Heitman, "Antifungal activities of antineoplastic agents: Saccharomyces cerevisiae as a model system to study drug action," Clinical Microbiology Reviews, vol. 12, no. 4, pp. 583-611, 1999.

[32] T. Edlind, L. Smith, K. Henry, S. Katiyar, and J. Nickels, "Antifungal activity in Saccharomyces cerevisiae is modulated by calcium signalling," Molecular Microbiology, vol. 46, no. 1, pp. 257-268, 2002.

[33] G. Serhan, C. M. Stack, G. G. Perrone, and C. O. Morton, "The polyene antifungals, amphotericin $\mathrm{B}$ and nystatin, cause cell death in Saccharomyces cerevisiae by a distinct mechanism to amphibian-derived antimicrobial peptides," Annals of Clinical Microbiology and Antimicrobials, vol. 13, p. 18, 2014.

[34] M. E. Cabral, L. I. Figueroa, and J. I. Farina, "Synergistic antifungal activity of statin-azole associations as witnessed by Saccharomyces cerevisiae- and Candida utilis-bioassays and ergosterol quantification," Revista Iberoamericana de Micología, vol. 30, no. 1, pp. 31-38, 2013.

[35] B. Tebbets, D. Stewart, S. Lawry et al., "Identification and characterization of antifungal compounds using a Saccharomyces cerevisiae reporter bioassay," PLoS One, vol. 7, no. 5, p. 4, 2012.

[36] D. Witters, K. Knez, F. Ceyssens, R. Puers, and J. Lammertyn, "Digital microfluidics-enabled single-molecule detection by printing and sealing single magnetic beads in femtoliter droplets," Lab on a Chip, vol. 13, no. 11, pp. 2047-2054, 2013.

[37] A. Gomes, E. Fernandes, and J. L. Lima, "Fluorescence probes used for detection of reactive oxygen species," Journal of Biochemical and Biophysical Methods, vol. 65, no. 2-3, pp. 45-80, 2005.

[38] M. M. Tarpey, D. A. Wink, and M. B. Grisham, "Methods for detection of reactive metabolites of oxygen and nitrogen: in vitro and in vivo considerations," American Journal of Physiology - Regulatory Integrative and Comparative Physiology, vol. 286, no. 3, pp. R431-R444, 2004.

[39] F. Madeo, E. Frohlich, and K. U. Frohlich, "A yeast mutant showing diagnostic markers of early and late apoptosis," The Journal of Cell Biology, vol. 139, no. 3, pp. 729-734, 1997.

[40] J. S. Beckman, T. W. Beckman, J. Chen, P. A. Marshall, and B. A. Freeman, "Apparent hydroxyl radical production by peroxynitrite: implications for endothelial injury from nitric oxide and superoxide," Proceedings of the National Academy of Sciences of the United States of America, vol. 87, no. 4, pp. 1620-1624, 1990.

[41] R. Radi, "Nitric oxide, oxidants, and protein tyrosine nitration," Proceedings of the National Academy of Sciences of the United States of America, vol. 101, no. 12, pp. 4003-4008, 2004.

[42] R. O. Poyton, K. A. Ball, and P. R. Castello, "Mitochondrial generation of free radicals and hypoxic signaling," Trends in Endocrinology and Metabolism, vol. 20, no. 7, pp. 332-340, 2009.

[43] D. D. Rees, R. M. Palmer, R. Schulz, H. F. Hodson, and S. Moncada, "Characterization of three inhibitors of endothelial nitric oxide synthase in vitro and in vivo," British Journal of Pharmacology, vol. 101, no. 3, pp. 746-752, 1990.

[44] L. Carmona, M. Gandia, B. Lopez-Garcia, and J. F. Marcos, "Sensitivity of Saccharomyces cerevisiae to the cell-penetrating antifungal peptide PAF26 correlates with endogenous nitric oxide (NO) production," Biochemical and Biophysical Research Communications, vol. 417, no. 1, pp. 56-61, 2012.

[45] N. S. Osorio, A. Carvalho, A. J. Almeida et al., "Nitric oxide signaling is disrupted in the yeast model for Batten disease," 
Molecular Biology of the Cell, vol. 18, no. 7, pp. 2755-2767, 2007.

[46] B. Almeida, S. Buttner, S. Ohlmeier et al., "NO-mediated apoptosis in yeast," Journal of Cell Science, vol. 120, Part 18, pp. 3279-3288, 2007.

[47] D. Collett, Modelling Survival Data in Medical Research, Chapman and Hall/CRC, 2 edition, 2003.

[48] T. V. M. Vila, K. Ishida, W. de Souza, K. Prousis, T. Calogeropoulou, and S. Rozental, "Effect of alkylphospholipids on Candida albicans biofilm formation and maturation," The Journal of Antimicrobial Chemotherapy, vol. 68, no. 1, pp. 113-125, 2013.

[49] E. Cantón, J. Pemán, A. Viudes, G. Quindós, M. Gobernado, and A. Espinel-Ingroff, "Minimum Fungicidal concentrations of amphotericin B for bloodstream Candida species," Diagnostic Microbiology and Infectious Disease, vol. 45, no. 3, pp. 203206, 2003.

[50] E. B. Cohen, "The role of signaling via aqueous pore formation in resistance responses to amphotericin B," Antimicrobial Agents and Chemotherapy, vol. 60, no. 9, pp. 5122-5129, 2016.

[51] E. Thomas, E. Roman, S. Claypool, N. Manzoor, J. Pla, and S. L. Panwar, "Mitochondria influence CDR1 efflux pump activity, Hog1-mediated oxidative stress pathway, iron homeostasis, and Ergosterol levels in Candida albicans," Antimicrobial Agents and Chemotherapy, vol. 57, no. 11, pp. 55555580, 2013.

[52] C. C. Uribe, F. Dos Santos de Oliveira, B. Grossmann et al., "Cytotoxic effect of amphotericin B in a myofibroblast cell line," Toxicology In Vitro, vol. 27, no. 7, pp. 2105-2109, 2013.

[53] L. Y. Chen, M. T. Sheu, C. K. Liao, F. C. Tsai, W. Y. Kao, and C. H. Su, "Taiwanofungus camphoratus (Syn Antrodia camphorata) extract and amphotericin B exert adjuvant effects via mitochondrial apoptotic pathway," Integrative Cancer Therapies, vol. 12, no. 2, pp. 153-164, 2013.

[54] I. Gusarov, K. Shatalin, M. Starodubtseva, and E. Nudler, "Endogenous nitric oxide protects bacteria against a wide spectrum of antibiotics," Science, vol. 325, no. 5946, pp. 1380-1384, 2009.

[55] T. Domitrovic, F. L. Palhano, C. Barja-Fidalgo, M. DeFreitas, M. T. Orlando, and P. M. Fernandes, "Role of nitric oxide in the response of Saccharomyces cerevisiae cells to heat shock and high hydrostatic pressure," FEMS Yeast Research, vol. 3, no. 4, pp. 341-346, 2003.

[56] R. Nasuno, M. Aitoku, Y. Manago, A. Nishimura, Y. Sasano, and H. Takagi, "Nitric oxide-mediated antioxidative mechanism in yeast through the activation of the transcription factor Mac1," PLoS One, vol. 9, no. 11, article e113788, 2014.

[57] R. I. Astuti, D. Watanabe, and H. Takagi, "Nitric oxide signaling and its role in oxidative stress response in Schizosaccharomyces pombe," Nitric Oxide, vol. 52, pp. 29-40, 2016.

[58] S. H. Qi, L. Y. Hao, J. Yue, Y. Y. Zong, and G. Y. Zhang, "Exogenous nitric oxide negatively regulates the S-nitrosylation p38 mitogen-activated protein kinase activation during cerebral ischaemia and reperfusion," Neuropathology and Applied Neurobiology, vol. 39, no. 3, pp. 284-297, 2013.

[59] J. Vitecek, A. Lojek, G. Valacchi, and L. Kubala, “Argininebased inhibitors of nitric oxide synthase: therapeutic potential and challenges," Mediators of Inflammation, vol. 2012, Article ID 318087, 22 pages, 2012.

[60] M. Ziche, L. Morbidelli, E. Masini et al., "Nitric oxide mediates angiogenesis in vivo and endothelial cell growth and migration in vitro promoted by substance P," The Journal of Clinical Investigation, vol. 94, no. 5, pp. 2036-2044, 1994.

[61] P. Kubes, M. Suzuki, and D. N. Granger, "Nitric oxide: an endogenous modulator of leukocyte adhesion," Proceedings of the National Academy of Sciences, vol. 88, no. 11, pp. 4651-4655, 1991.

[62] M. Sonntag, A. Deussen, and J. Schrader, "Role of nitric oxide in local blood flow control in the anaesthetized dog," Pflügers Archiv, vol. 420, no. 2, pp. 194-199, 1992.

[63] G. A. Van Buren, D. S. Yang, and K. E. Clark, "Estrogen-induced uterine vasodilatation is antagonized by Lnitroarginine methyl ester, an inhibitor of nitric oxide synthesis," American Journal of Obstetrics and Gynecology, vol. 167, no. 3, pp. 828-833, 1992.

[64] A. Vials and G. Burnstock, "Effects of nitric oxide synthase inhibitors, L-NG-nitroarginine and L-NG-nitroarginine methyl ester, on responses to vasodilators of the guinea-pig coronary vasculature," British Journal of Pharmacology, vol. 107, no. 2, pp. 604-609, 1992.

[65] R. G. Kilbourn, C. Szabo, and D. L. Traber, "Beneficial versus detrimental effects of nitric oxide synthase inhibitors in circulatory shock: lessons learned from experimental and clinical studies," Shock, vol. 7, no. 4, pp. 235-246, 1997.

[66] A. Petros, D. Bennett, and P. Vallance, "Effect of nitric oxide synthase inhibitors on hypotension in patients with septic shock," The Lancet, vol. 338, no. 8782-8783, pp. 1557-1558, 1991.

[67] J. A. Avontuur, R. P. Tutein Nolthenius, J. W. van Bodegom, and H. A. Bruining, "Prolonged inhibition of nitric oxide synthesis in severe septic shock: a clinical study," Critical Care Medicine, vol. 26, no. 4, pp. 660-667, 1998.

[68] D. H. Yates, S. A. Kharitonov, P. S. Thomas, and P. J. Barnes, "Endogenous nitric oxide is decreased in asthmatic patients by an inhibitor of inducible nitric oxide synthase," American Journal of Respiratory and Critical Care Medicine, vol. 154, no. 1, pp. 247-250, 1996.

[69] J. M. Wecht, J. P. Weir, A. H. Krothe, A. M. Spungen, and W. A. Bauman, "Normalization of supine blood pressure after nitric oxide synthase inhibition in persons with tetraplegia," The Journal of Spinal Cord Medicine, vol. 30, no. 1, pp. 5-9, 2007.

[70] J. M. Wecht, J. P. Weir, D. S. Goldstein et al., "Direct and reflexive effects of nitric oxide synthase inhibition on blood pressure," American Journal of Physiology Heart and Circulatory Physiology, vol. 294, no. 1, pp. H190-H197, 2008.

[71] J. M. Wecht, M. Radulovic, D. Rosado-Rivera, R. L. Zhang, M. F. LaFountaine, and W. A. Bauman, "Orthostatic effects of midodrine versus L-NAME on cerebral blood flow and the renin-angiotensin-aldosterone system in tetraplegia," Archives of Physical Medicine and Rehabilitation, vol. 92, no. 11, pp. 1789-1795, 2011.

[72] B. Chudzik, M. Koselski, A. Czurylo, K. Trebacz, and M. Gagos, "A new look at the antibiotic amphotericin B effect on Candida albicans plasma membrane permeability and cell viability functions," European Biophysics Journal, vol. 44, no. 1-2, pp. 77-90, 2015.

[73] T. J. Walsh, V. Yeldandi, M. McEvoy et al., "Safety, tolerance, and pharmacokinetics of a small unilamellar liposomal formulation of amphotericin B (AmBisome) in neutropenic patients," Antimicrobial Agents and Chemotherapy, vol. 42, pp. 2391-2398, 1998. 


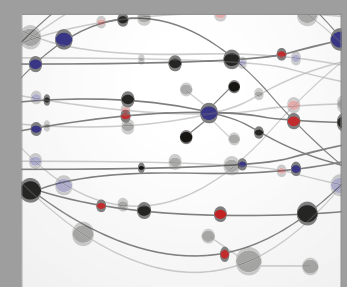

The Scientific World Journal
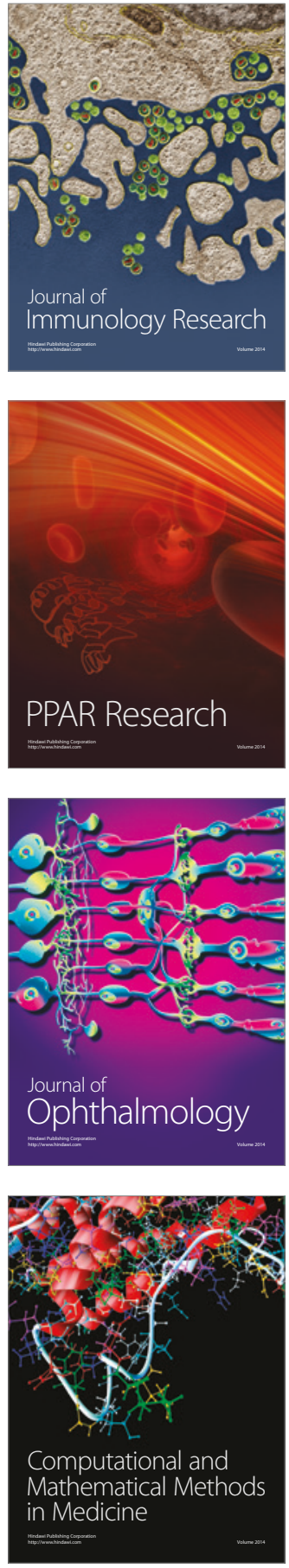

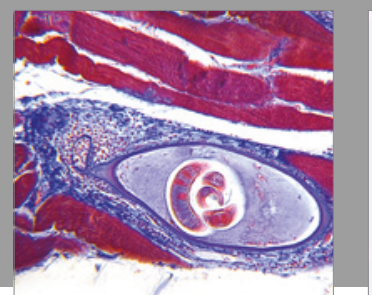

Gastroenterology Research and Practice
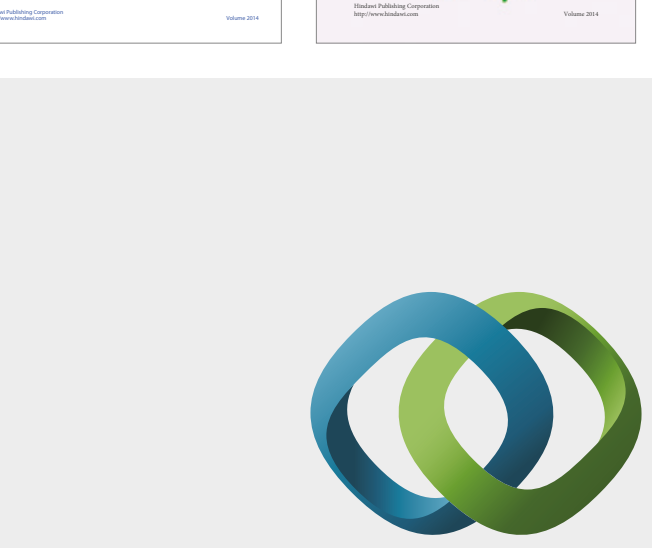

\section{Hindawi}

Submit your manuscripts at

https://www.hindawi.com
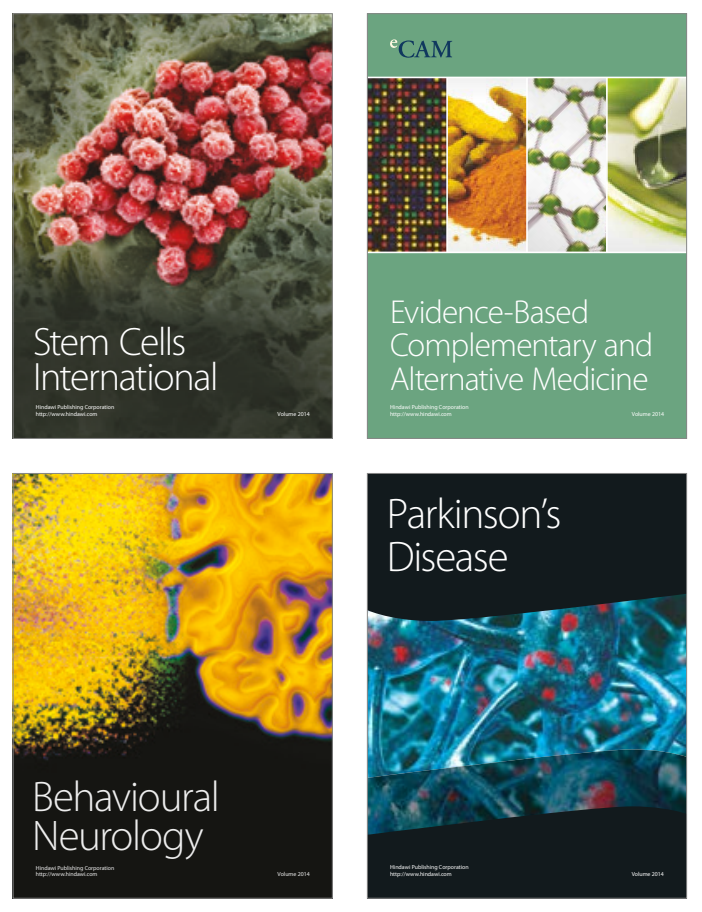
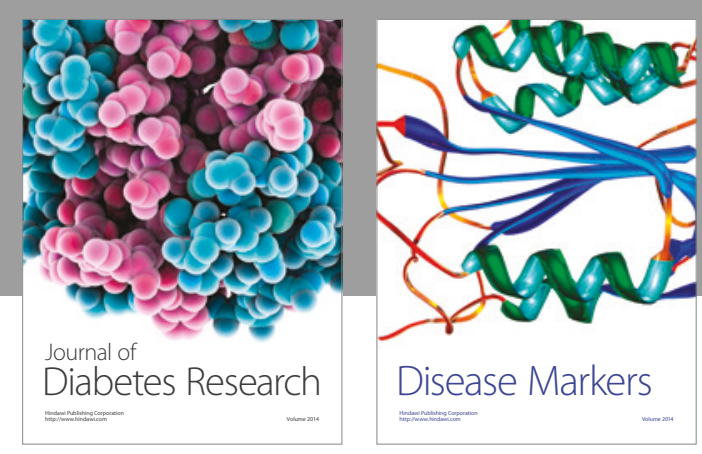

Disease Markers
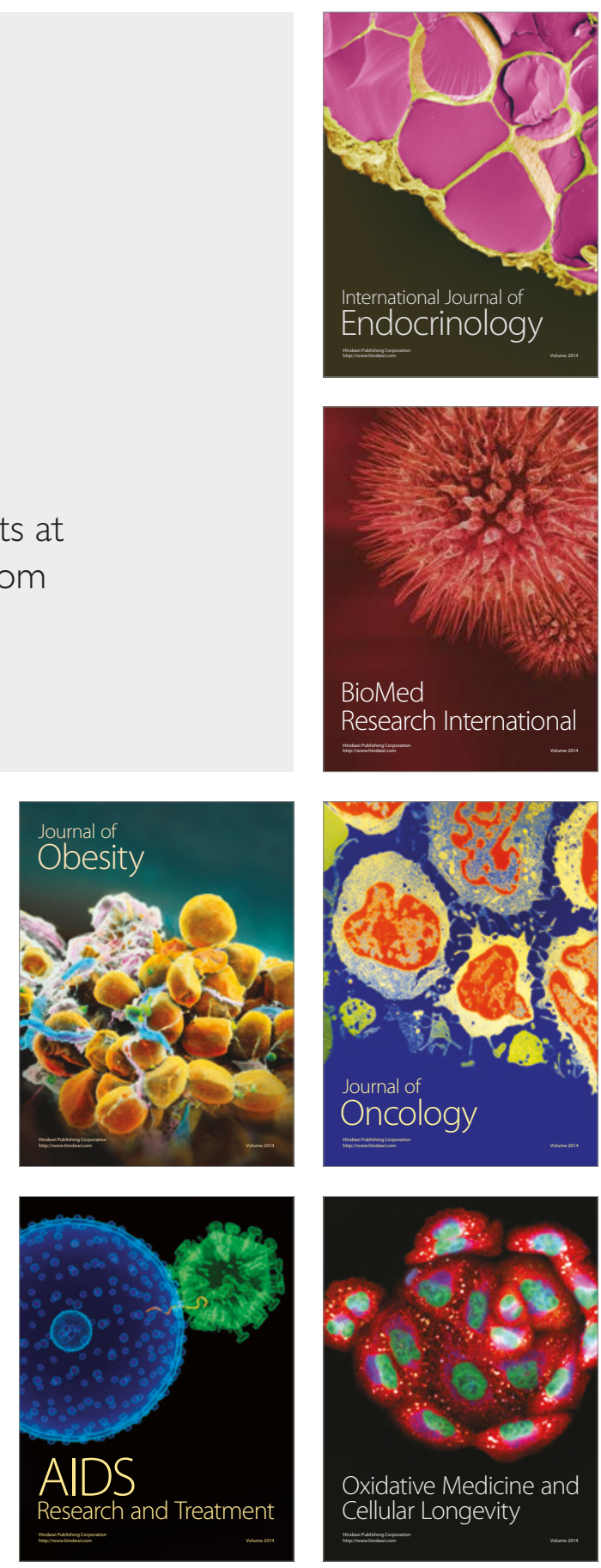